\title{
Dynamics and energy spectra of aperiodic discrete-time quantum walks
}

\author{
N. Lo Gullo, ${ }^{1}$ C. V. Ambarish, ${ }^{2}$ Th. Busch, ${ }^{3}$ L. Dell'Anna, ${ }^{4}$ and C. M. Chandrashekar ${ }^{2,5}$ \\ ${ }^{1}$ Department of Physics, Univeritá degli Studi di Milano, Via G. Celoria, 16, 20133 Milano, Italy \\ ${ }^{2}$ The Institute of Mathematical Sciences, C. I. T. Campus, Taramani, Chennai 600113, India \\ ${ }^{3}$ Okinawa Institute of Science and Technology Graduate University, 904-0495 Okinawa, Japan \\ ${ }^{4}$ Department of Physics and Astronomy, Universitá degli Studi di Padova, Via Marzolo, 8, 35131 Padova, Italy \\ ${ }^{5}$ Homi Bhabha National Institute, Training School Complex, Anushakti Nagar, Mumbai 400094, India
}

(Received 18 November 2016; revised manuscript received 16 May 2017; published 6 July 2017)

\begin{abstract}
We investigate the role of different aperiodic sequences in the dynamics of single quantum particles in discrete space and time. For this we consider three aperiodic sequences, namely, the Fibonacci, Thue-Morse, and RudinShapiro sequences, as examples of tilings the diffraction spectra of which have pure point, singular continuous, and absolutely continuous support, respectively. Our interest is to understand how the order, intrinsically introduced by the deterministic rule used to generate the aperiodic sequences, is reflected in the dynamical properties of the quantum system. For this system we consider a single particle undergoing a discrete-time quantum walk (DTQW), where the aperiodic sequences are used to distribute the coin operations at different lattice positions (inhomogeneous DTQW) or by applying the same coin operation at all lattice sites at a given time but choosing different coin operation at each time step according to the chosen aperiodic sequence (time dependent DTQW). We study the energy spectra and the spreading of an initially localized wave packet for different cases, finding that in the case of Fibonacci and Thue-Morse tilings the system is superdiffusive, whereas in the Rudin-Shapiro case it is strongly subdiffusive. Trying to understand this behavior in terms of the energy spectra, we look at the survival amplitude as a function of time. By means of the echo we present strong evidence that, although the three orderings are very different as evidenced by their diffraction spectra, the energy spectra are all singular continuous except for the inhomogeneous DTQW with the Rudin-Shapiro sequence where it is discrete. This is in agreement with the observed strong localization both in real space and in the Hilbert space. Our paper is particularly interesting because quantum walks can be engineered in laboratories by means of ultracold gases or in optical waveguides, and therefore would be a perfect playground to study singular continuous energy spectra in a completely controlled quantum setup.
\end{abstract}

DOI: 10.1103/PhysRevE.96.012111

\section{INTRODUCTION}

Quantum walks are the quantum-mechanical analog of classical random walks and their dynamics can be engineered in various quantum systems. Just like for their classical counterparts, two variants exist: discrete-time quantum walks (DTQWs) [1] and continuous-time quantum walks (CTQWs) [2]. They have played an important role in the field of quantum information processing as a powerful component of quantum algorithms [3,4] and have been shown to be a universal quantum computation primitive in that any quantum computation can be realized efficiently [5,6]. Furthermore, with the ability to engineer quantum walks by controlling the parameters of the evolution operators, quantum effects such as localization [7,8] and topological bound states [9] have been simulated. Controlled dynamics of quantum walks have also allowed the simulation of relativistic quantum dynamics where the speed of light is mimicked by the parameter in the evolution operator [10-14]. Their versatility and ease of implementation in physical systems such as NMR [15,16], trapped ions $[17,18]$, atoms $[19,20]$, and photonic systems $[21,22]$ make them promising candidates to explore and engineer quantum dynamics beyond the Schrödinger equation. Among the two variants of quantum walks, DTQWs are defined for a particle with an internal degree of freedom, to which the quantum coin operations are applied. These quantum coin operations can be position and time dependent, which can lead to deviations from quadratic spreading of the wave packet [23], including the emergence of Anderson localization [24] for a completely disordered arrangement of coin operations in position space and/or time [7,25]. It is therefore natural to wonder about the behavior of DTQWs when the coin operation is aperiodic in nature [26]. Studies of the behavior of DTQWs for coin operations that are arranged in the aperiodic Fibonacci sequence [26] have already found a diffusive behavior without any signature of localization $[27,28]$. However, whether this behavior is limited to just this sequence or extends to other aperiodic sequences as well is not yet clear and we will address this question here.

Below we will first briefly introduce the deterministic aperiodic sequences that we will study and that have previously been of great interest in condensed matter physics, photonics, and material sciences $[29,30]$.

Materials with deterministic aperiodic sequences can be thought of as in between materials with long-range translational symmetry (crystals) and amorphous materials with no long range order. Quasicrystals are one example [31] and searching for other nonperiodic materials that have a crystal-like spectrum is an active research area $[32,33]$. Of particular interest in this field have been structures based on the Fibonacci sequence [34,35], the Thue-Morse sequence [36], and the Rudin-Shapiro sequence [37]. The Fibonacci quasicrystal has been studied in great detail because it has a pure point spectrum [34,35]. Thue-Morse crystals were shown to have a singular continuous spectrum [36] and the 
\begin{tabular}{l|lllll|l|l|l|l|l|l|l|l|l|l} 
A & B & A & B & A & B & A & B & A & B & A & B & A & B & A & Periodic
\end{tabular} \begin{tabular}{lllll|l|l|l|l|l|l|l|l|l|l|l|l} 
A & B & A & A & B & A & B & A & A & B & A & A & B & A & B & Fibonacci
\end{tabular}

\begin{tabular}{ll|l|l|l|l|l|l|l|l|l|l|l|l|l|l|l} 
A & B & B & A & B & A & A & B & B & A & A & B & A & B & B & Thue-Morse \\
\hline
\end{tabular}

\section{\begin{tabular}{|l|l|l|l|l|l|l|l|l|l|l|l|l|l|l|l} 
A & A & A & B & A & A & B & A & A & A & A & B & B & B & A & Rudin-Shapiro \\
\hline
\end{tabular}}

FIG. 1. Schematic of the arrangement of the two values for the periodic and the deterministic aperiodic sequences.

Rudin-Shapiro crystals have a fully continuous spectrum [37]. Several measures have been proposed to quantify the amount of order in aperiodic sequences, for instance based on entropy $[38,39], \log$ entropy [40], and the structure of the energy spectrum [41]. In Fig. 1 we show a schematic of the different aperiodic sequences.

Here we study the dynamics of the DTQW with spatial and temporal dislocations in the coin operator based on the three deterministically aperiodic sequences mentioned above: the quasiperiodic Fibonacci sequence, the critical Thue-Morse sequence, and the Rudin-Shapiro sequence. We compare our results with the ones for a standard two-periodic sequence of the form $\mathrm{ABAB}$... and show that the support of the diffraction pattern plays a crucial role in determining the spreading properties of the quantum walker. By studying their energy spectra and their asymptotic behavior we show that the DTQW using the Fibonacci sequence, which has a pure point spectrum, shows diffusive spreading behavior similar to the periodic walks. The Rudin-Shapiro sequence, which has a continuous spectrum, shows prominent localization behavior which is closer to a walk with a random coin sequence. Quantum walks using the Thue-Morse sequence with a singular continuous spectrum comprise both diffusing and localized components in the dynamics.

The paper is organized as follows. In Sec. II we briefly review the DTQW and in Sec. III we introduce the different sequences of aperiodic walks with position and time dependent coin operations. In Secs. IV and V we present the study of energy spectra and the spreading of the walk for all configurations. In Sec. VI we present the asymptotic behavior and conclude in Sec. VII.

\section{DISCRETE-TIME QUANTUM WALK}

The one-dimensional DTQW is defined on a position space and an internal (coin) space. The position space is spanned by the basis vectors $|x\rangle$, with $x \in \mathbb{Z}$, and the internal space of the particle is a two-dimensional space spanned by $|\uparrow\rangle=\left(\begin{array}{l}1 \\ 0\end{array}\right)$ and $|\downarrow\rangle=\left(\begin{array}{l}0 \\ 1\end{array}\right)$. Each step of the walk consists of the application of one coin and one shift operator. The coin operator is unitary and can be written as

$$
\hat{C}(\theta)=\left(\begin{array}{cc}
\cos (\theta) & -\sin (\theta) \\
\sin (\theta) & \cos (\theta)
\end{array}\right) .
$$

The shift operator performs the translation in the position space conditioned on the internal degree of freedom of the walker and can be written as

$$
\hat{S}=\sum_{x=-N}^{N}|\uparrow\rangle\langle\uparrow|\otimes| x-1\rangle\langle x|+| \downarrow\rangle\langle\downarrow|\otimes| x+1\rangle\langle x| .
$$

When the coin operation is homogeneous and time independent, the state of the system after $t$ steps $(t \in \mathbb{N})$ is given by $\left|\psi_{t}\right\rangle=\hat{W}(\theta)\left|\psi_{t-1}\right\rangle$ where $\hat{W}(\theta)=\hat{S}[\hat{I} \otimes \hat{C}(\theta)]$. In the following we will consider the situation where the coin operator can have two values, $\theta_{1}$ and $\theta_{2}$, and is either time dependent but homogeneous in space or inhomogeneous in space and constant in time.

\section{APERIODIC QUANTUM WALKS WITH POSITION AND TIME DEPENDENT COIN OPERATIONS}

In this section we will first introduce the sequences of the coin operations for the aperiodic settings we are going to study and then calculate the spread in the probability distribution after a certain time $t$. As a reference, we will also consider a DTQW with a regular distribution of two coins, which we will call a period sequence henceforth. For all walks we will fix $\theta_{1}=\pi / 4$ and study the dynamics for different values of $\theta_{2}$. Other choices of $\theta_{1}$ do not qualitatively change the results we obtain.

The support of the diffraction pattern of the chosen sequence plays a crucial role in determining the spreading properties of the walker. According to the Lebesgue theory of measure the support can be decomposed into pure point, singular continuous, and absolutely continuous components. The Fibonacci, Thue-Morse, and Rudin-Shapiro quasicrystals are particularly well suited to study the role of each of these components, since their diffraction measures have pure point, singular continuous, and absolutely continuous support, respectively. On the other hand it has also been shown that their energy spectra are all singular continuous [41,42]. Our aim is to investigate the implications of the nature of the energy spectra on the dynamical properties of the quantum system.

\section{A. Periodic and aperiodic sequences}

\section{Periodic sequence}

The two-period sequence is of the form $\mathrm{ABABAB} \ldots \mathrm{AB}$ and it is the base sequence we will use to compare the deterministic aperiodic sequences to.

\section{Fibonacci sequence}

The Fibonacci sequence of two elements is defined by the substitution rule $A \rightarrow A B$ and $B \rightarrow A$, which can recursively be written as $S_{N+1}=S_{N} S_{N-1}\left(S_{1}=B, S_{2}=A\right)$.

\section{Thue-Morse sequence}

The Thue-Morse sequence of two elements is defined by the substitution rule $A \rightarrow A B$ and $B \rightarrow B A$ or recursively as $S_{N+1}=S_{N} \overline{S_{N}}\left(S_{1}=A\right)$, where $\overline{S_{N}}$ is the string $S_{N}$ in which the two letters $A$ and $B$ have been exchanged.

\section{Rudin-Shapiro sequence}

The Rudin-Shapiro sequence is defined by a four-element substitution sequence with the rules given by $P \rightarrow P Q, Q \rightarrow$ 
$P R, R \rightarrow S Q$, and $S \rightarrow S R$. To obtain a sequence composed of only two elements, A and B, we perform the mapping $(P, Q) \rightarrow A,(R, S) \rightarrow B$

\section{B. Position dependent coin operations}

In order to define the position dependent coin operations we will label each spatial site either $A$ or $B$, starting with $A$ at the extreme left position $(-N)$ and increasing by one unit until the final position on the right hand side $(N)$ is reached. For example, the word sequence $A B A A B$ will correspond to the lattice the site indices of which are $-2,-1,0,1,2$ and the coin operation at each site will be performed accordingly by the replacement $A \rightarrow \theta_{1}$ and $B \rightarrow \theta_{2}$. By defining the states $\left|\nearrow_{x}\right\rangle=\hat{C}[\theta(x)]|\uparrow\rangle$ and $|\swarrow x\rangle=\hat{C}[\theta(x)]|\downarrow\rangle$, we can rewrite the single step evolution operator $\hat{W}=\hat{S} \hat{C}^{\prime}$ as

$$
\hat{W}=\sum_{x=-N}^{N}|\uparrow\rangle\left\langle\nearrow_{x}|\otimes| x-1\right\rangle\langle x|+| \downarrow\rangle\left\langle\swarrow_{x}|\otimes| x+1\right\rangle\langle x| .
$$

This allows us to interpret the evolution of the system as an itinerant spin in an inhomogeneous magnetic field and we expect the mobility of the spin to be related to the spatial structure of the chosen sequence. To see this let us consider the single step evolution operator $\hat{W}=\hat{S} \otimes \hat{C}^{\prime}$ where

$$
\begin{aligned}
\hat{S} & =\sum_{x=-N}^{N}|\uparrow\rangle\langle\uparrow|\otimes| x-1\rangle\langle x|+| \downarrow\rangle\langle\downarrow|\otimes| x+1\rangle\langle x|, \\
\hat{C}^{\prime} & =\sum_{x=-N}^{N}|x\rangle\langle x| \otimes \hat{C}[\theta(x)] .
\end{aligned}
$$

Because $\theta(x)$ can take only two values, $\theta_{1}$ or $\theta_{2}$, we can partition the whole lattice $\mathcal{L}$ into two sublattices $\mathcal{L}_{1}$ and $\mathcal{L}_{2}$ such that $\mathcal{L}=\mathcal{L}_{1} \cup \mathcal{L}_{2}$ where $\mathcal{L}_{i}=\left\{x: \theta(x)=\theta_{i}\right\}$.

Using this observation we can rewrite

$$
\hat{C}^{\prime}=\sum_{x=-N}^{N}|x\rangle\langle x| \otimes\left[w(x) \hat{C}_{1}+\bar{w}(x) \hat{C}_{2}\right],
$$

where $\hat{C}_{i}=\hat{C}\left(\theta_{i}\right), \quad w(x)=1 \quad \forall x \in \mathcal{L}_{1}, \quad$ and $\bar{w}(x)=1-$ $w(x)=1 \quad \forall x \in \mathcal{L}_{2}$. The function $w(x)[\bar{w}(x)]$ encodes information on the geometry of the original spatial distribution of the two coins regardless of the actual form of the coin operators. Once expressed in this form it is clear that the dynamics of the walker is linked to the underlying geometric distribution of the letters $A$ and $B$ of the sequence used. To see this let us introduce the states $|k\rangle$ such that $\langle x \mid k\rangle=e^{-l x k} / \sqrt{L}$ with $L=2 N+1$ and the identity (for the spatial part) in this basis being $\hat{\mathbf{1}}=\int d k|k\rangle\langle k|$. This allows us to write

$$
\hat{C}=\int d k d q|k\rangle\langle q| \otimes\left[f(k-q) \hat{C}_{1}+\bar{f}(k-q) \hat{C}_{2}\right]
$$

where

$$
\begin{aligned}
& f(q)=\frac{1}{L} \sum_{x=-N}^{N} e^{l q x} w(x), \\
& \bar{f}(q)=\frac{1}{L} \sum_{x=-N}^{N} e^{l q x} \bar{w}(x) .
\end{aligned}
$$

The Fourier amplitudes $\tilde{f}(q)$ are nothing but the amplitudes of the diffraction spectrum of the weighted Dirac combs $\omega=$ $\sum_{x} w(x) \delta_{x}$ and its reverse $\bar{\omega}=\sum_{x} \bar{w}(x) \delta_{x}$.

As we shall see, the effect of changing $\theta_{2}$ is to change the diffusion coefficient of the walker, but not the way the walker diffuses through the lattice (except for some special values such as $\theta_{2}=\pi / 4,3 \pi / 4$ and $\theta_{2}= \pm \pi / 2$ which will be discussed separately in the following).

\section{Time dependent coin operations}

To study the effect of temporally dependent coin operation we consider the application of $\hat{W}[\theta(t)]$ at the $t$ th time step where $\theta(t)$ is chosen according to the distribution of $A \rightarrow \theta_{1}$ and $B \rightarrow \theta_{2}$ in the chosen sequence. For instance, if the chosen string is $A B A B B B A A B B$, then after $t=6$ time steps the evolution operator will be $\hat{U}=$ $\hat{W}\left(\theta_{2}\right) \hat{W}\left(\theta_{2}\right) \hat{W}\left(\theta_{2}\right) \hat{W}\left(\theta_{1}\right) \hat{W}\left(\theta_{2}\right) \hat{W}\left(\theta_{1}\right)$.

Similar to the case of the position dependent coin we can define states $\left|\nearrow_{t}\right\rangle=\hat{C}[\theta(t)]|\uparrow\rangle$ and $\left|\swarrow_{t}\right\rangle=\hat{C}[\theta(t)]|\downarrow\rangle$, which allows us to rewrite the single step evolution operator as

$$
\begin{aligned}
\hat{W}[\theta(t)]= & \sum_{x=-N}^{N}|\uparrow\rangle\left\langle\nearrow_{t}|\otimes| x-1\right\rangle\langle x|+| \downarrow\rangle \\
& \times\langle\swarrow t|\otimes| x+1\rangle\langle x| .
\end{aligned}
$$

The total evolution operator connecting the initial state to the state at time step $t$, namely, $\hat{U}(t)=\prod_{s=1}^{t} \hat{W}[\theta(s)]$, can be interpreted as the evolution of a spin in a time dependent magnetic field. In this sense the change in coin operation for each time (change in magnetic field) in the system can be interpreted as a periodically kicked itinerant spin on a lattice with a kick amplitude depending upon the chosen aperiodic sequence.

\section{ENERGY SPECTRA}

In this section we discuss the energy spectra for the DTQWs using the different sequences introduced in the preceding section. Here a comment is necessary on what we mean by energy spectrum in this context and how we derive it. In general an operator of the form given in Eq. (3) acting on a finite space is not a unitary operator due to the spatial part. The iterative application of the single step operators therefore results, in general, in a nonprobability conserving evolution under a total operator $\hat{T}$. The latter is such that $\hat{T} \hat{T}^{\dagger}=\hat{T}^{\dagger} \hat{T}=\hat{I}$ still acts as a unitary operator in a sublattice $[-\tilde{N}, \tilde{N}]$ with $\tilde{N}<N$. Therefore, for localized initial states and for a certain $t^{*}$ such that the probability $p_{x}=\left|\left\langle x, \uparrow \mid \psi\left(t^{*}\right)\right\rangle\right|^{2}+\left|\left\langle x, \downarrow \mid \psi\left(t^{*}\right)\right\rangle\right|^{2}$ of finding the walker at a given position $x$ is $p_{x}=0$ if $x \notin[-\tilde{N}, \tilde{N}]$, we can restrict the dynamics of the walker 


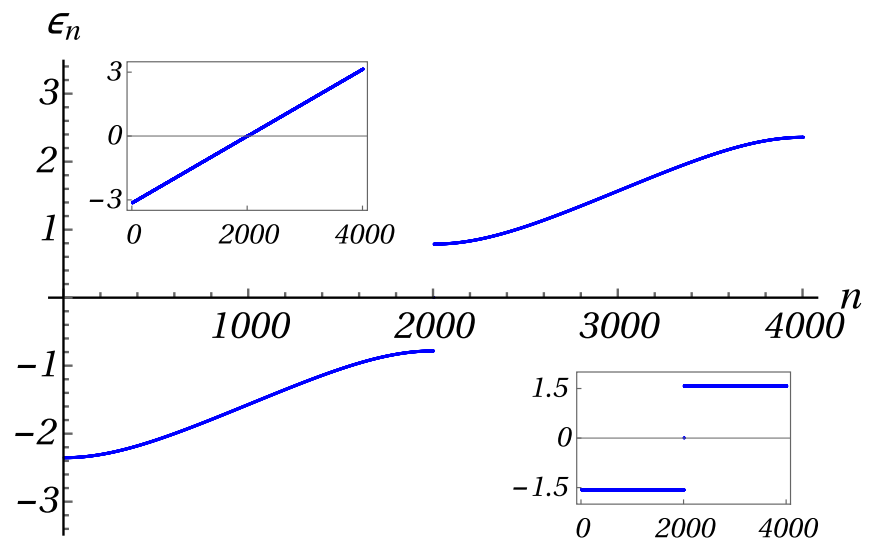

FIG. 2. Spectrum $\epsilon_{n}=\imath \log \left(\lambda_{n}\right)$ where $\lambda_{n}$ are the eigenvalues of the operator $\hat{W}$ in Eq. (3) for the case $\theta_{1}=\theta_{2}=\pi / 4$. Energy spectra $\epsilon_{n}=\imath \log \left(\lambda_{n}\right)$ where $\lambda_{n}$ are the eigenvalues of the operator $\hat{W}(\theta)$ in Eq. (3), for $\theta=0$ (left) and $\theta=\pi / 2$ (right). Insets: Spectrum for the case $\theta_{1}=-\theta_{2}=\pi$ (top left) and $\theta_{1}=-\theta_{2}=\pi / 2$ (bottom right).

to that interval and consider it as a unitary evolution. The energy spectrum is then the spectrum of the unitary operator $\hat{U}$ which is the restriction of $\hat{T}$ acting onto the above mentioned subspace. It is worth stressing that this is mainly due to the numerical limitations, whereas this problem does not arise in the thermodynamic limit. In what follows we will set $N=2 t$, where $t$ is the number of time steps and we check that the probability of finding the walker ten sites away from the boundaries is exactly zero at the chosen final time.

The energy spectrum of a homogeneous and time independent DTQW for the case $\theta=\pi / 4$ is shown in Fig. 2. It is given by $\epsilon_{n}=\imath \log \left(\lambda_{n}\right)$, with the $\lambda_{n}$ being the eigenvalues of $\hat{W}(\theta)$, and can be seen to consist of two main bands, which is what one would expect from a tight binding Hamiltonian with a two-element unit cell.

The two bands are due to the presence of the internal degree of freedom (coin) and two extreme cases are (a) $\theta=x \pi$ and (b) $\theta=(2 x-1) \pi / 2$ which correspond to $\hat{C}=(-1)^{x} \hat{I}$ and $(-1)^{x}(|\uparrow\rangle\langle\downarrow|-| \downarrow\rangle\langle\uparrow|)$, respectively. To see this let us consider an infinite lattice $(N \rightarrow \infty)$ and introduce the states $|k\rangle$ such that $\langle x \mid k\rangle=e^{-l x k} / \sqrt{L}$ with $L=2 N+1$ and the identity (for the spatial part) in this basis $\hat{\mathbf{1}}=\int d k|k\rangle\langle k|$. This allows us to rewrite the single step evolution operator as

$$
\hat{W}=\int d k|k\rangle\langle k| \otimes\left(e^{-\imath k}|\uparrow\rangle\left\langle\uparrow\left|+e^{\imath k}\right| \downarrow\right\rangle\langle\downarrow|\right) \hat{C}(\theta) .
$$

For the case (a) $C(x \pi)= \pm \hat{I}_{C}$ the eigenstates of $\hat{W}$ are $|k\rangle|\uparrow\rangle$ and $|k\rangle|\downarrow\rangle$ with eigenvalues $e^{-l k}$ and $e^{\imath k}$, respectively. On the other hand in case (b) $\hat{C}=(-1)^{x}(|\uparrow\rangle\langle\downarrow|-| \downarrow\rangle\langle\uparrow|)$ the eigenstates are given by $e^{ \pm l \frac{\pi}{2}} \int d k|k\rangle\left(e^{l \frac{k}{2}}|\uparrow\rangle \pm e^{-l \frac{k}{2}}|\downarrow\rangle\right) / \sqrt{2}$ with eigenvalues $\pi / 2$ and $-\pi / 2$. Therefore the spectrum in case (a) is linear without a gap at $\epsilon=0$ whereas in case (b) it is flat inside the two bands which are separated by a gap $\Delta=\pi$ as shown in the insets of Fig. 2.

When it comes to the study of the energy spectra of DTQWs the distinction between spatial aperiodic sequences and temporal aperiodic ones is important.
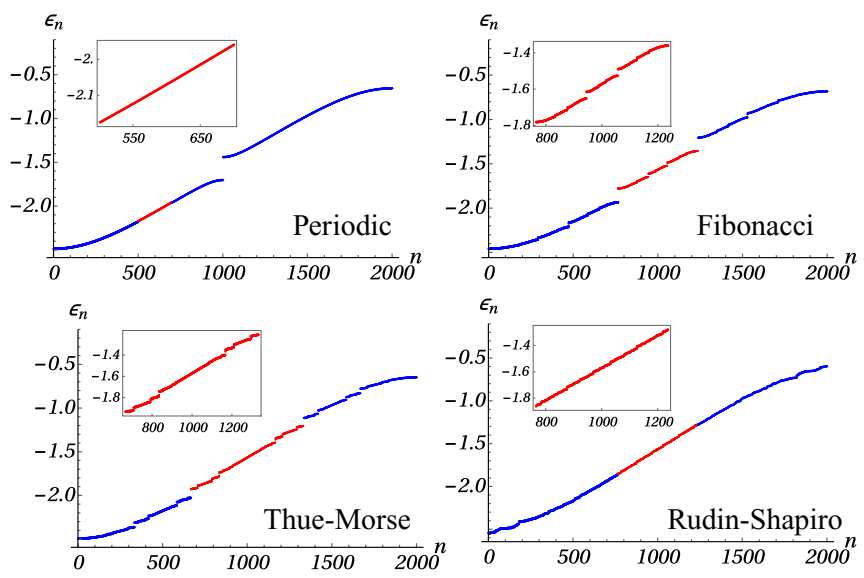

FIG. 3. Spectrum $\epsilon_{n}=\imath \log \left(\lambda_{n}\right)$ where the $\lambda_{n}$ are the eigenvalues of the operator $\hat{W}(\theta)$ in Eq. (3). The angles chosen are $\theta_{1}=\pi / 4$ and $\theta_{2}=\pi / 6$ and are distributed (from top left to bottom right) according to the periodic, the Fibonacci, the Thue-Morse, and the Rudin-Shapiro sequence. In the inset of each figure we show a zoomed in part of the spectrum to show the self-similarity of the spectra and the presence of gaps at all energies.

\section{A. Position dependent coin operations}

In the case of DTQWs with position dependent coin operations $\left(\theta_{2} \neq \theta_{1}\right)$ distributed according to a given aperiodic sequence the spectrum of the system is the same at all time steps and coincides with that of the single step evolution operator $\hat{W}(\theta)$. It can be seen from Fig. 3 that the effect of different coin operations on different sites is to open new gaps inside the two above mentioned main energy bands, thus creating sub-bands. The position of these new gaps can be determined in first order perturbation theory (i.e., considering the case $\left|\theta_{2}-\theta_{1}\right| \approx 0$ ) by finding that they open where the intensity of the Fourier transform of $w(x)$ is higher [41-44]. Within the two main bands the structure is therefore determined by the spatial distribution of the two coins and consequently by the geometry of the chosen arrangement.

The above spectra give rise to the densities of states (DOS) shown in Fig. 4, from which key properties of each distribution can be identified. In particular we notice the self-similarity of the energy spectrum of the Fibonacci sequence (top right) and the broad availability of states for the Rudin-Shapiro sequence throughout the whole sub-band. We also notice that each DOS for all aperiodic arrangements shows singular features (spikes in the DOS), which are a result of the opening of gaps in the energy spectrum on a set of zero measure $[41,42]$. This translates into plateaus in the integrated DOS.

\section{B. Time dependent coin operations}

In the case of DTQWs with time dependent coin operations given by aperiodic sequences we deal with a nonautonomous quantum system and therefore we cannot, strictly speaking, define a general energy spectrum. However, we can look at the instantaneous or the asymptotic energy spectrum. Applying different coin operations at different time steps amounts to changing the energy spectrum between two possible spectra 

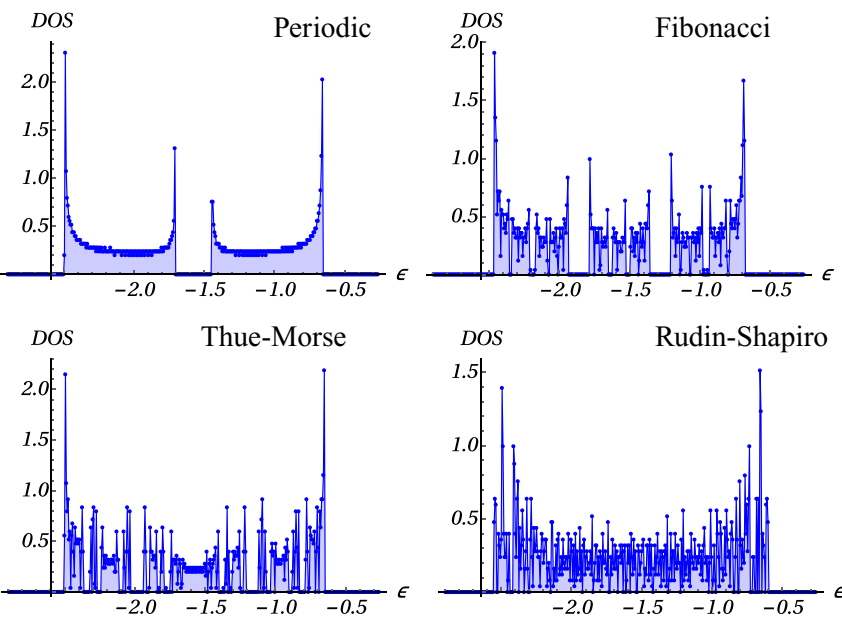

FIG. 4. Density of states normalized to the total number of states corresponding to the spectra in Fig. 3. The energy window used to compute these graphs is the same as the one that will be used for the analysis of the asymptotic properties $d \epsilon=2 \pi / t_{f}$ where $t_{f}=1000$ is the number of steps.

in an aperiodic way and the two instantaneous energy spectra for the cases $\theta_{1}=\pi / 2$ and $\theta_{2}=\pi / 6$ are shown in Fig. 5 .

To determine the asymptotic properties of the system it is necessary to look at the spectrum of the total evolution operator $\hat{U}(t)$ for $t \gg 1$. From a numerical analysis we note that after only a few $(t \approx 30)$ steps the spectrum of the total evolution operator does not appreciably change any more. The results for the different sequences are shown in Fig. 6 and one can immediately notice the absence of sub-band gaps, which characterized the spectra in the spatially dependent case. On the other hand, the DOS for the spectrum of the asymptotic evolution operator reveals a much richer structure (see Fig. 7) than what we could have gathered from the spectrum itself. In particular one can note features of the self-similarity for the Fibonacci (top right) and Thue-Morse (bottom left) cases. This implies that, although the coin distribution is completely homogeneous across the system at each time step, the aperiodic

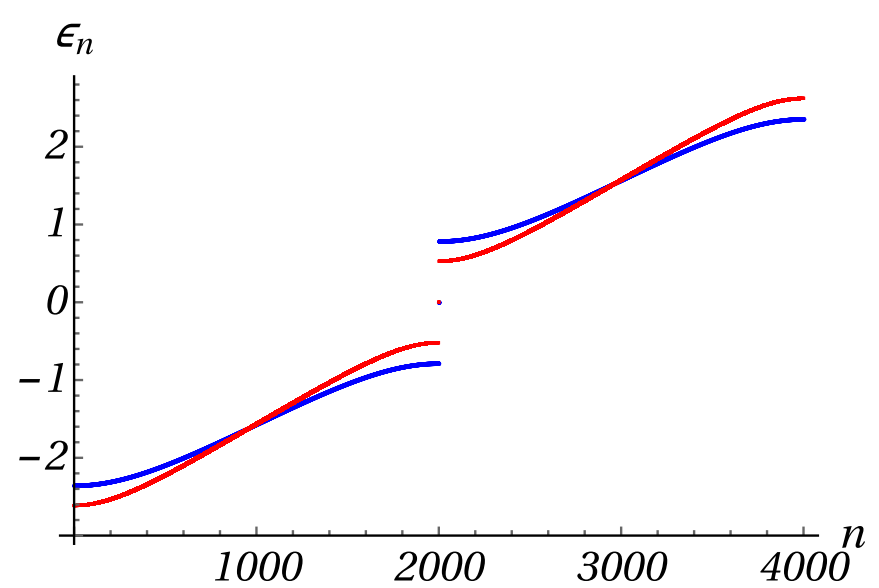

FIG. 5. Spectrum $\epsilon_{n}=\imath \log \left(\lambda_{n}\right)$ where $\lambda_{n}$ are the eigenvalues of the operator $\hat{W}\left(\theta_{1}\right)$ (blue) and $W\left(\theta_{2}\right)$ (red) for the case $\theta_{1}=\pi / 4$ and $\theta_{2}=\pi / 6$.
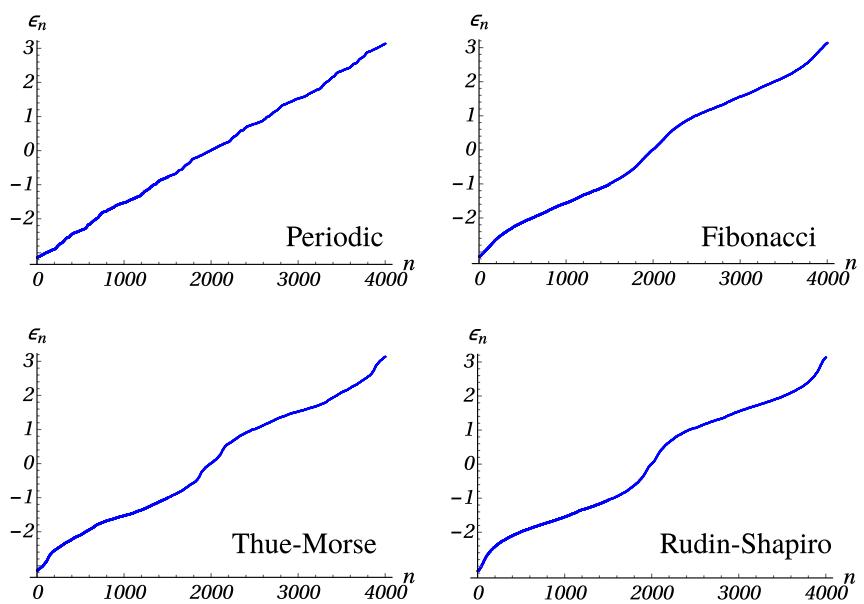

FIG. 6. Lowest band of the spectrum $\epsilon_{n}=\imath \log \left(\lambda_{n}\right)$ where $\lambda_{n}$ are the eigenvalues of the operator $\hat{U}(t)$ with $t=500$. The angles chosen are $\theta_{1}=\pi / 4$ and $\theta_{2}=\pi / 6$.

nature of the temporal distribution arises clearly in the long time limit.

\section{SPREADING OF THE WALKER}

In order to characterize the dynamics of the walker we look at both the mean displacement $\langle x(t)\rangle$ and its variance given by $\sigma^{2}(t)=\left\langle x^{2}(t)\right\rangle-\langle x(t)\rangle^{2}$. We choose the initial state to be $|\psi\rangle=\left|x_{0}\right\rangle \otimes \frac{|\uparrow\rangle+i|\downarrow\rangle}{\sqrt{2}}$, such that the spatial part is initially localized at site $x_{0}$. In the following we will discuss the different sequences in detail.

\section{A. Periodic and aperiodic sequences}

\section{Periodic sequence}

The dynamics of a standard DTQW with a single, fixed coin operator is known to be always ballistic, with a velocity
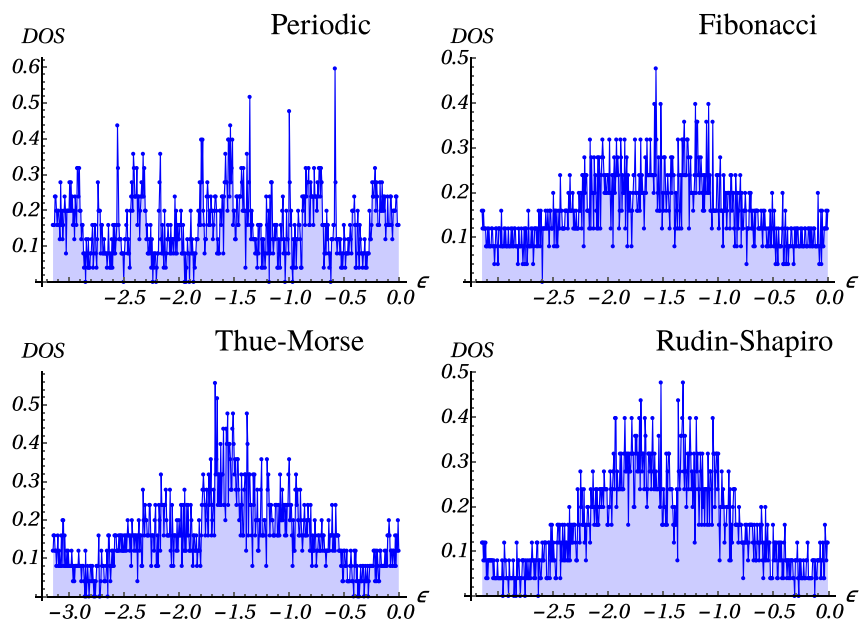

FIG. 7. Densities of states normalized to the total number of states and corresponding to the spectra shown in Fig. 6. The energy window used to compute it is the same as the one that will be used for the analysis of the asymptotic properties $d \epsilon=2 \pi / t_{f}$ where $t_{f}=500$ is the number of steps. 

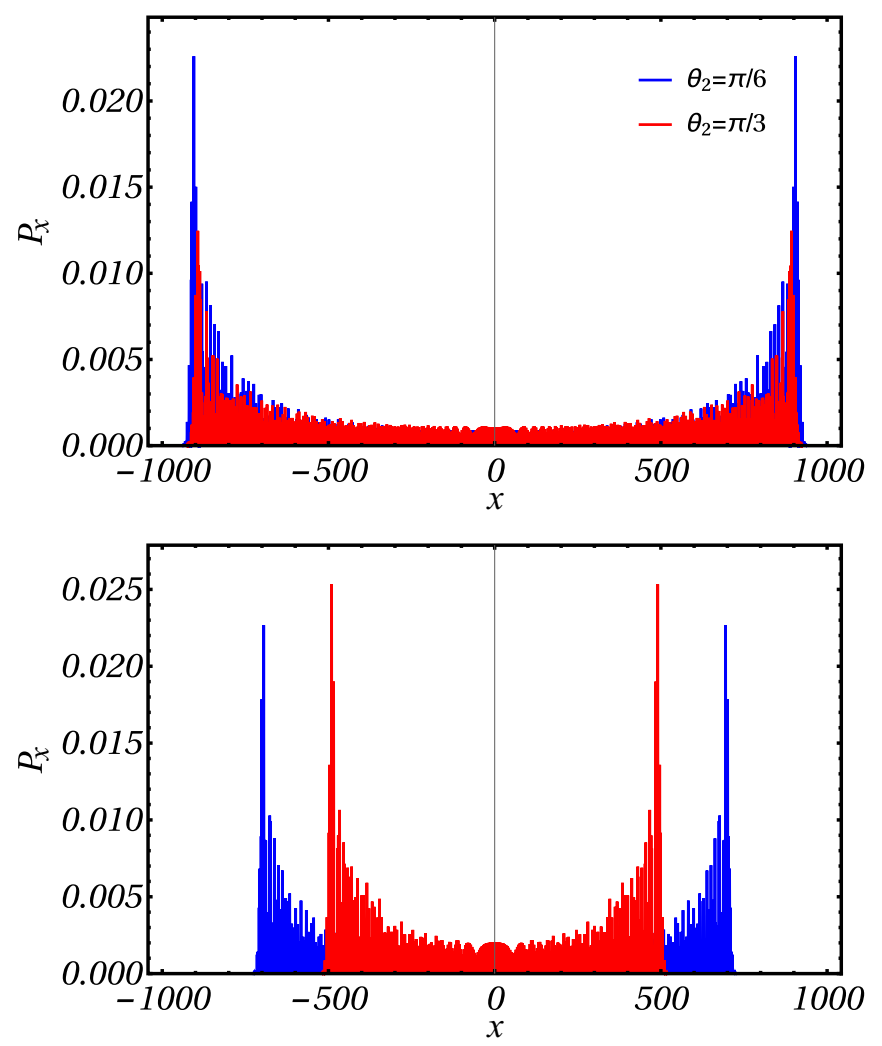

FIG. 8. Probability distribution after 1000 steps of DTQW using a two-periodic coin for two sets of angles $\left(\theta_{1}, \theta_{2}\right)$ with same $\theta_{1}=\pi / 4$. The probability distribution with spatial coin dependence and temporal coin dependence is shown in top and bottom figures, respectively. (Same parameters for other plots of walks).

that depends on $\theta$. From Fig. 8 one can see that this property prevails for the periodic sequence in the spatial as well as in the temporal case, but with the velocity $v$ depending now on $\theta_{1}$ and $\theta_{2}$. This result is not very surprising, since the presence of a periodicity means that localization is absent and therefore the spread in position space has to have a form that has the characteristic two-peaks structure of the standard DTQW.

\section{Fibonacci sequence}

The probability distributions in position space after 500 steps with the spatial or temporal arrangement of the coin operation given by the Fibonacci sequence are shown in Fig. 9. It is immediately notable that neither walk features the peaks in the outer regions and that no strongly localized peak at origin $(x=0)$ exists. This indicates that the Fibonacci sequence leads to DTQWs with diffusive behavior. We will analyze and characterize the nature of this diffusive behavior in detail below.

\section{Thue-Morse sequence}

The probability distributions for this sequence are shown in Fig. 10. In the spatial and the temporal case a prominent peak localized at $x=0$ can be seen, while at the same time a diffusive component is visible as well.
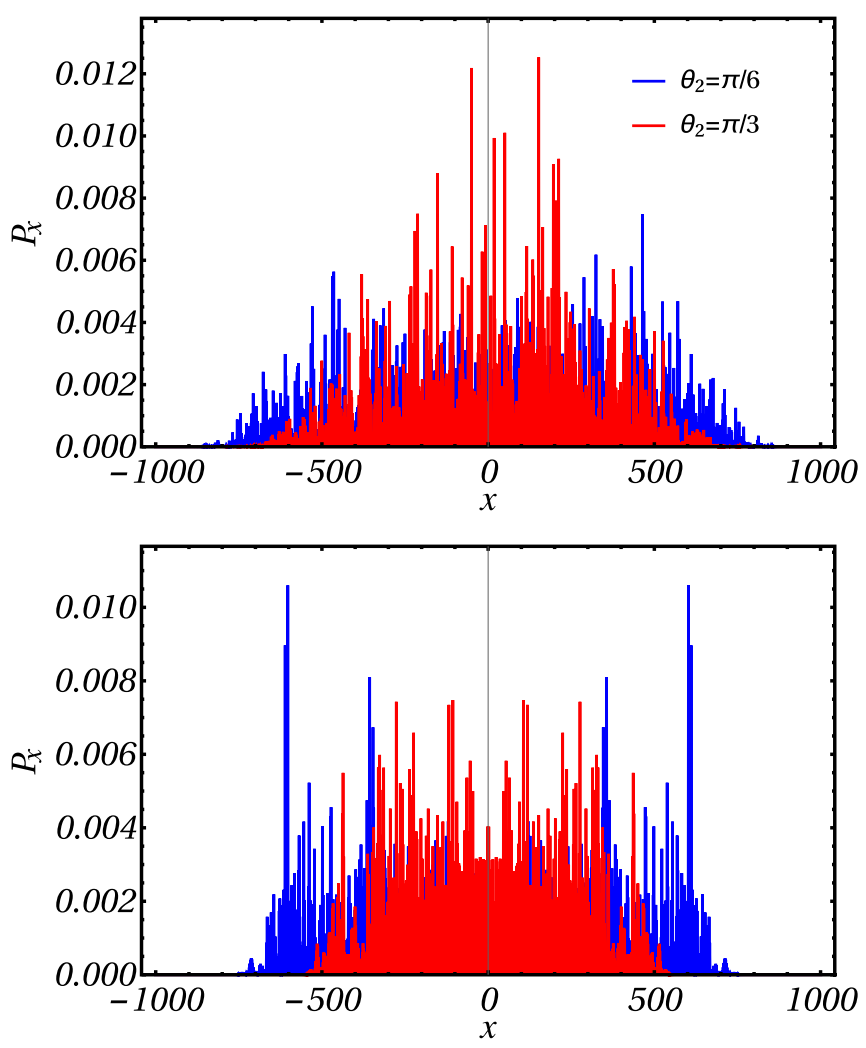

FIG. 9. Same as Fig. 8, but using a Fibonacci coin. For both situations the distribution can be seen to be dispersive.
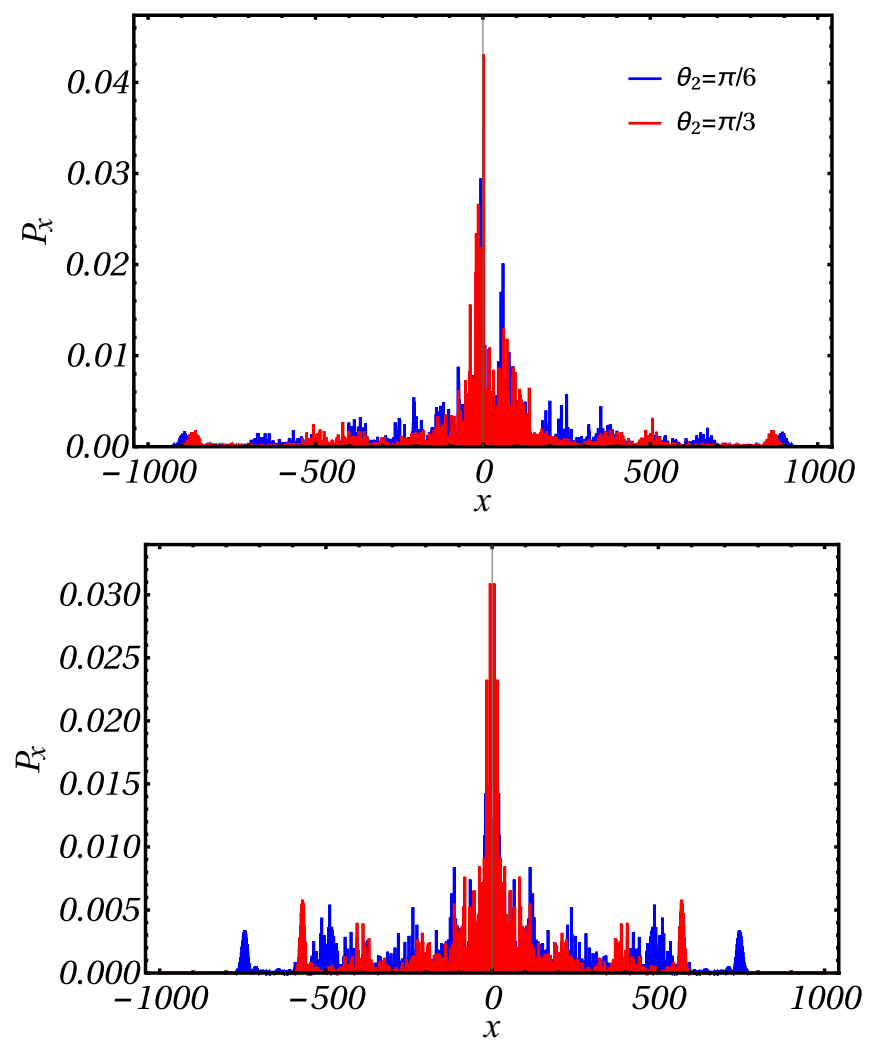

FIG. 10. Same as Fig. 8, but using a Thue-Morse coin. For both situations a localized and a diffusing component are visible in the distribution. 

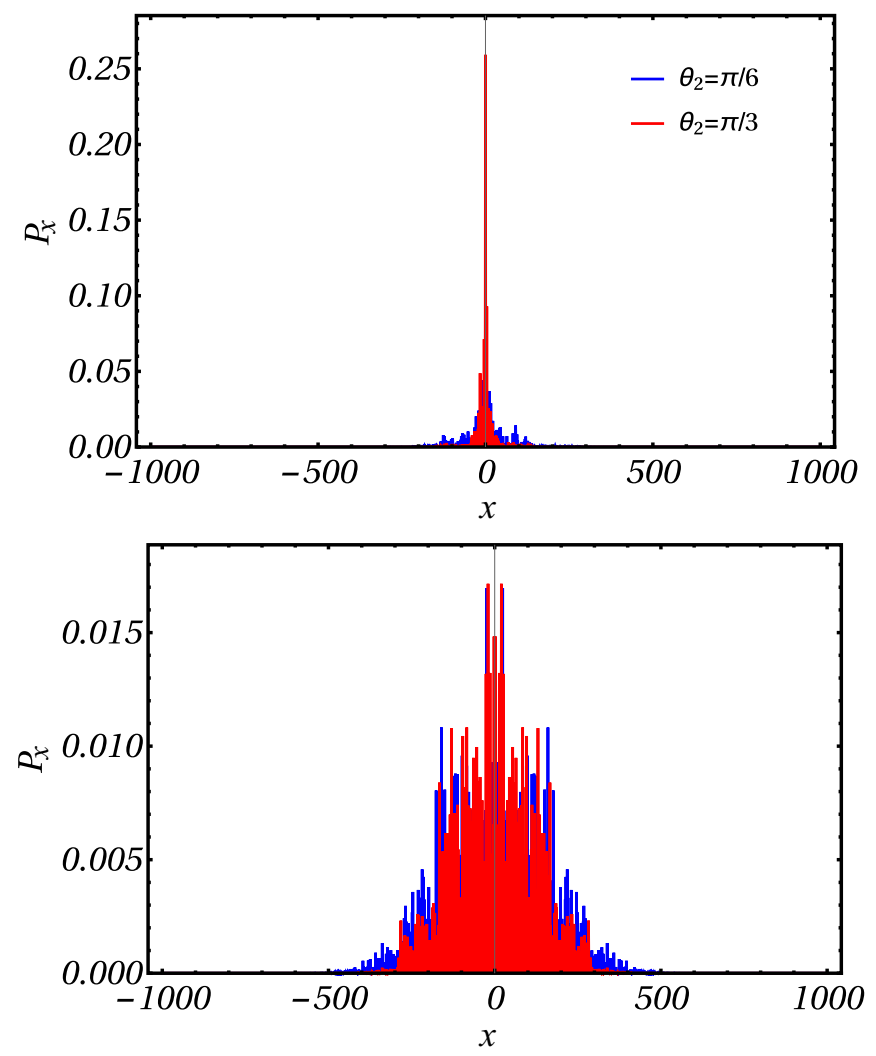

FIG. 11. Same as Fig. 8, but using a Rudin-Shapiro coin. The distribution using the spatial sequence is more strongly localized around the origin than the one for the temporal sequence.

\section{Rudin-Shapiro sequence}

The probability distributions for this sequence are shown in Fig. 11. One can see that, compared to the spread for the Fibonacci and the Thue-Morse sequences, the probability distribution for the Rudin-Shapiro sequence is more localized around the origin, $x=0$. The localization is also stronger for the spatial sequence than for the temporal sequence.

To better understand the different dynamics displayed by the aperiodic walks, we will characterize the dynamics as a function of time and link the behavior to the energy spectra of these walks in more detail below.

\section{B. Position dependent coin operations}

We will first focus on the walks with spatially varying coins and note that while in the standard DTQW evolution the spread of the probability distribution depends on the coin parameter $\theta[23,45]$ its general form is independent of the specific value. We can therefore restrict the parameter space by choosing one of the coins to be a Hadamard $\left(\theta_{1}=\pi / 4\right)$ and the obtained results will hold for all values of $\theta_{1}$.

Let us first look at the Fibonacci sequence, which has a pure point spectrum and is therefore the closest to a periodic structure. Comparing Figs. 8(a) and 9(a) one can see that the width of the spread in position space is roughly identical for both. However, the standard deviations shown in Fig. 12 show a slower increase for the Fibonacci sequence compared to the periodic sequence. In fact, the difference between them grows
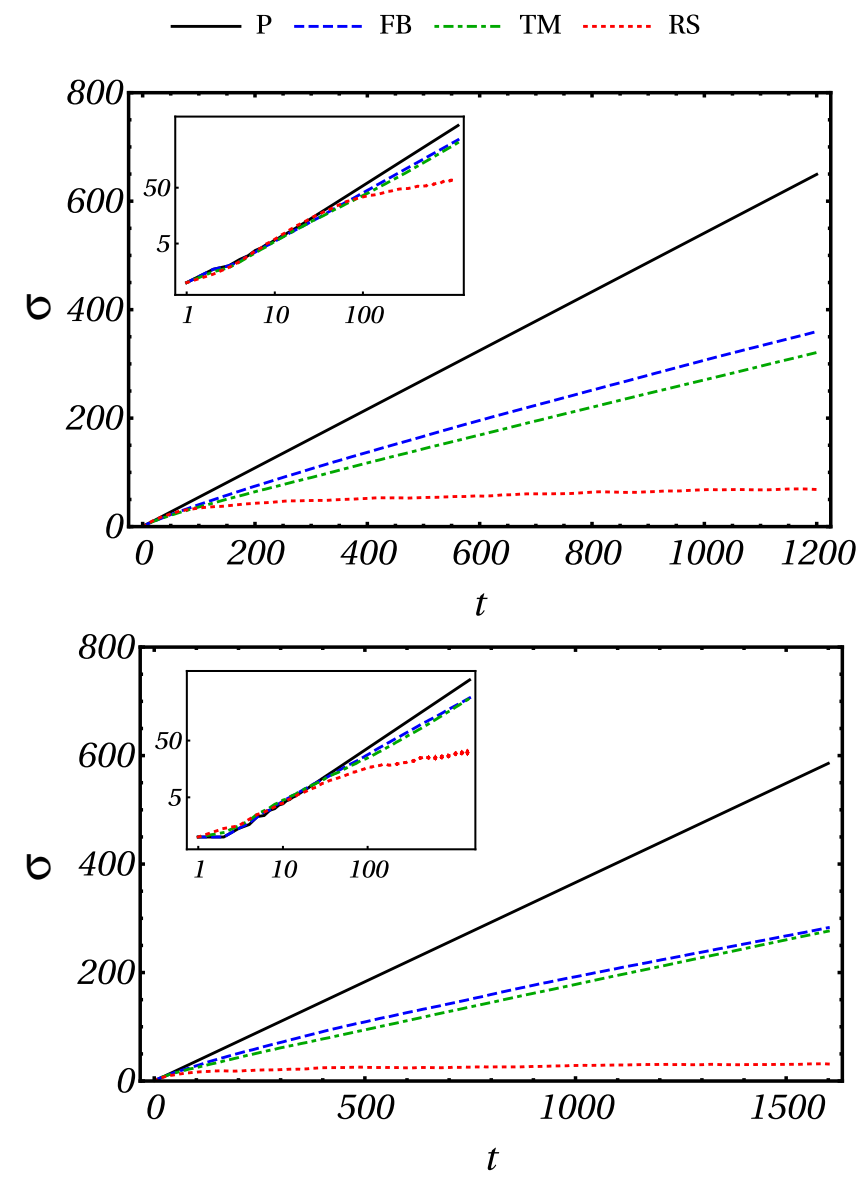

FIG. 12. Standard deviation after 1000 steps using inhomogeneous coins for a walker initially localized at the center of the lattice $x_{0}=0$ and $L=2001$. Top: $\theta_{2}=\pi / 6$. Bottom: $\theta_{2}=\pi / 3$. The notations used for the sequences are $\mathrm{P} \leftrightarrow$ Periodic, FB $\leftrightarrow$ Fibonacci, TM $\leftrightarrow$ Thue-Morse, and RS $\leftrightarrow$ Rudin-Shapiro. Insets: Double logarithmic scale plots showing the power-law dependence $\sigma(t) \propto t^{\alpha}$.

with increasing number of steps. Unlike the periodic walk, where the peaks in the probability distribution are observed at the outer edges of the distribution, the distribution from the Fibonacci sequence shows larger peaks at multiple spatial positions.

For the walk using the Thue-Morse sequence, which has a singular continuous spectrum, the probability distribution in Fig. 10 shows that the distribution has both a localized and a diffusing component. The standard deviation as function of number of steps shown in Fig. 12 shows that it is smaller than the periodic sequence and the Fibonacci sequence.

Finally, the Rudin-Shapiro sequence has an absolutely continuous diffraction spectrum, similar to a completely disordered medium. Here by disorder we mean a random distribution of two values assigned to each point on the lattice. From Figs. 11 and 12 one can see that the distribution localizes around the origin and the value of the standard deviation remains small with increasing number of steps.

It is also important to note that $\sigma(t)$ has a power-law dependence on $t$, which implies that $\sigma(t) \approx D t^{\alpha}$. This can be used to give a more systematic classification of the spreading 

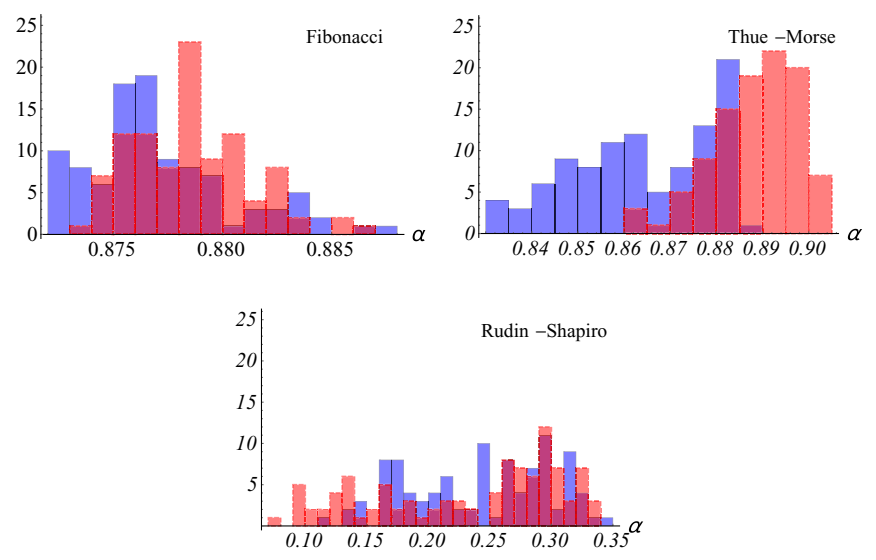

FIG. 13. Distribution of the fitting parameter $\alpha$ describing the long-time behavior of $\sigma(t) \propto t^{\alpha}$, for different initial positions of the walker on the lattice, for the inhomogeneous quantum walk with $\theta_{1}=\pi / 4$ and $\theta_{2}=\pi / 6$. The blue (red) histogram refers to a system size of $L=1001$ (2001).

properties for each sequence in the asymptotic limit and in the following we will show that the Fibonacci and ThueMorse sequences display superdiffusive behavior $(\alpha>1 / 2)$ whereas the Rudin-Shapiro sequence is strongly subdiffusive $(\alpha<1 / 2)$. To do this we first need to rule out that the aperiodic nature of the considered sequences introduces effects due to the particular choice of the initial position of the walker on the lattice. For instance, in the Fibonacci case, one can have three different types of configuration for a given site, namely, $A A, A B$, and $B A$. To investigate this possible dependence we have considered evolutions starting from all possible different initial states, and calculated for each of these cases the time dependent $\sigma(t)$ and the exponent $\alpha$. These calculations have confirmed that, for long times, $\alpha$ is independent of the choice of the initial site of the walker (within an error) and in Fig. 13 we show the distribution of $\alpha$ for a system with 1001 sites starting from 101 different initial positions in an interval with center around $x_{0}=0$ (the center of the lattice) and for $\theta_{1}=\pi / 4$, $\theta_{2}=\pi / 6$. From these distributions it is possible to get the mean values

$$
\begin{gathered}
\alpha_{\mathrm{FB}}=(0.877 \pm 0.003) \quad \text { Fibonacci } \\
\alpha_{\mathrm{TM}}=(0.86 \pm 0.01) \quad \text { Thue-Morse } \\
\alpha_{\mathrm{RS}}=(0.24 \pm 0.05) \quad \text { Rudin-Shapiro. }
\end{gathered}
$$

This allows us to conclude that, within the estimated standard deviation, the Fibonacci and the Thue-Morse sequences lead to a superdiffusive behavior, whereas the spreading of the wave packet for the Rudin-Shapiro sequence is greatly suppressed, showing a clear subdiffusive behavior. On the other hand, one can expect that the value of $\alpha$ also depends on the particular angle $\theta_{2}$ as already visible in Fig. 12 . We therefore repeated the above analysis for different values of $\theta_{2}$ and show in Fig. 14 the mean of the distribution over 101 different initial positions around the center of the lattice as a function of $\theta_{2}$ with the error bars given by the standard deviation of the distribution of points.
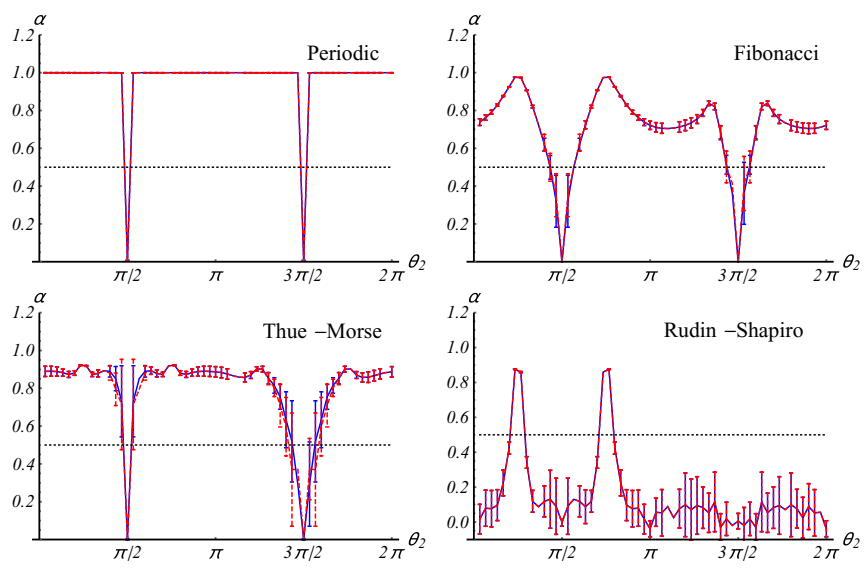

FIG. 14. Dependence of the exponent $\alpha$ (see main text) upon $\theta_{2}$ for inhomogeneous DTQWs with $\theta_{1}=\pi / 4$. Each point and the relative error bar are obtained by choosing 101 different initial positions around the center of the lattice $x=0$ and for $L=1001$ (solid blue line) and $L=2001$ (dashed red line). The dotted horizontal black line corresponds to $\alpha=1 / 2$ (diffusive case).

From these results we can conclude that the Fibonacci and the Thue-Morse sequences induce a superdiffusive behavior for the walker whereas the Rudin-Shapiro sequence shows a subdiffusive one for all values of $\theta_{2}$. However, special cases exist at $\theta_{2}=\pi / 4,3 \pi / 4$ and $\theta_{2}= \pm \pi / 2$. In the first case $\left(\theta_{2}=\pi / 4,3 \pi / 4\right)$ one recovers a homogeneous distribution of the coin operations across the lattice and therefore a purely ballistic $(\alpha=1)$ behavior is obtained. On the other hand in the second case $\left(\theta_{2}= \pm \pi / 2\right)$ the second coin operation amounts to flipping the internal degrees of freedom of the coin (up to a phase factor, namely, $\hat{C}(\pi / 2)=\mp l \hat{\sigma}_{y}$ ) and therefore at each site where $\hat{C}\left(\theta_{2}\right)$ acts the walker experiences a "mirroring" effect (the walker reverses its motion), resulting in a suppression of the spreading.

\section{Time dependent coin operations}

The time dependent case holds particular interest because, as was shown in Sec. IV, the spectrum of the asymptotic evolution operator apparently loses the properties of the aperiodic sequences chosen and no connection between the sequence itself and the dynamical properties of the system seems to exist. Despite this, we will show in the following that the dynamical properties of the system are strongly influenced by the chosen sequence and fingerprints of each of them are left in the spreading properties of the walker.

The standard deviations of a DTQW with time dependent coin operations after 1000 steps for two particular values of $\theta_{2}$ $\left(\theta_{1}=\pi / 4\right)$ are shown in Fig. 15. One can see that the standard deviation is in general lowest for the Rudin-Shapiro sequence, which has the absolutely continuous diffraction spectrum that resembles the properties of a disordered distribution. This is similar to the inhomogeneous case discussed above, however in the time dependent case the spreading is larger. The Fibonacci and the Thue-Morse sequences induce very fast diffusion through the lattice, which is comparable to the periodic one. This suggests that the signatures of the three sequences are still clearly present in the spreading properties 

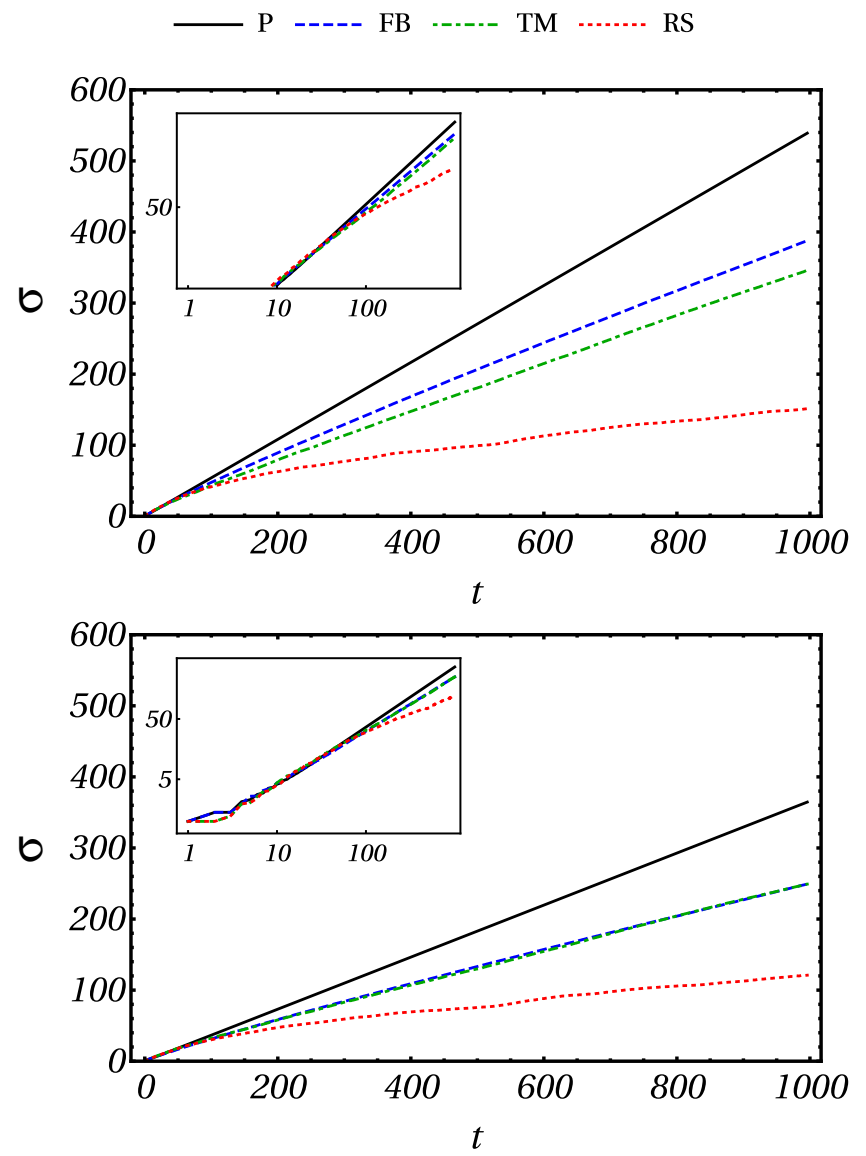

FIG. 15. Standard deviation for time dependent coin for a walker initially localized at the center of the lattice $x_{0}=0$ and $L=2001$. Top: $\theta_{2}=\pi / 6$. Bottom: $\theta_{2}=\pi / 3$. The notations used for the sequences are $\mathrm{P} \leftrightarrow$ Periodic, FB $\leftrightarrow$ Fibonacci, TM $\leftrightarrow$ Thue-Morse, and RS $\leftrightarrow$ Rudin-Shapiro. Insets: Double logarithmic scale plots showing the power-law dependence $\sigma(t) \propto t^{\alpha}$.

although the asymptotic energy spectra of the evolution operator did not show clearly identifiable properties, as they did in the inhomogeneous case. Furthermore, even in this case the asymptotic behavior of $\sigma(t)$ seems to show a power-law dependence upon $t$.

In Fig. 16 we show the dependence of the exponent $\alpha$ as a function of $\theta_{2}$ in order to determine the spreading behavior of the walker. There are two main observations one can make: (i) the Thue-Morse sequence induces a very robust, with respect to a variation of $\theta_{2}$, superdiffusive behavior, even stronger than in the spatial case; and (ii) the Rudin-Shapiro sequence induces a nearly diffusive behavior (not-anomalous $\alpha \approx 1 / 2$ ), unlike the strongly subdiffusive behavior observed in the inhomogeneous DTQW.

\section{ASYMPTOTIC BEHAVIOR}

We now turn to the characterization of the asymptotic properties of the DTQWs with different coin sequences and will highlight the relation between the asymptotic behavior of the walker distribution and the spectral properties of the chosen aperiodic sequence. In order to make this discussion quantitative we will choose the so-called survival probability
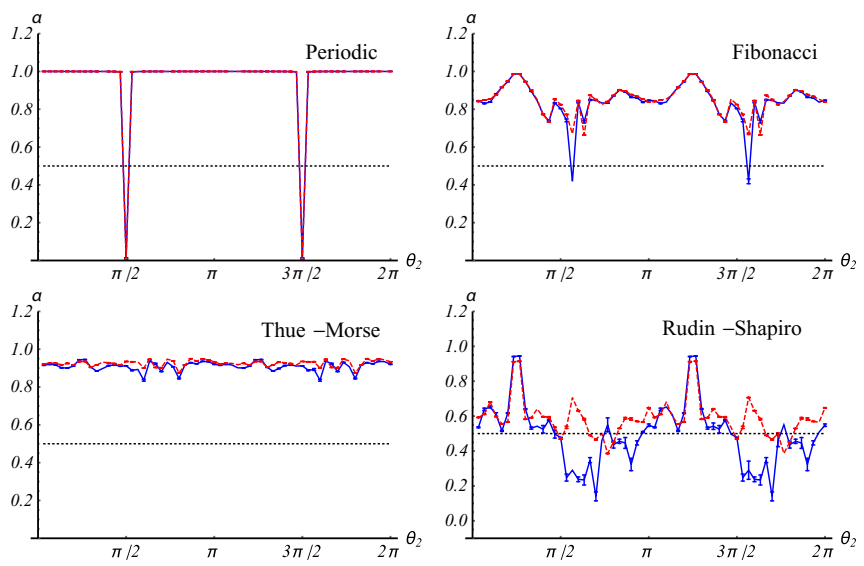

FIG. 16. Dependence of the exponent $\alpha$ (see main text) upon $\theta_{2}$ for time dependent DTQWs with $\theta_{1}=\pi / 4$. Each point and the relative error bar are obtained by choosing 101 different initial positions as in the inhomogeneous case (although in this case it would not be necessary if we considered periodic boundary conditions, therefore enforcing translational invariance) around the center of the lattice $x=0$ and for $L=1001$ (solid blue line) and $L=2001$ (dashed red line). The dotted horizontal black line corresponds to $\alpha=1 / 2$ (diffusive case).

or Loschmidt echo $\mathcal{L}(t)=|v(t)|^{2}$ as our figure of merit, which is defined via its amplitude:

$$
v(t)=\langle\psi(0) \mid \psi(t)\rangle,
$$

where $|\psi(t)\rangle=U(t)|\psi(0)\rangle$.

The reason to consider this quantity is twofold. On one hand it has a clear physical meaning because it describes the probability of finding the evolved state in the initial state after some time $t$. Therefore, it is strictly linked to the study of ergodicity and localization of a quantum system and as such has been widely studied in statistical mechanics. On the other hand it is directly related to the Fourier transform of the spectral measure of the evolution operator [46] (and therefore, for an autonomous system, of its infinitesimal generator, the Hamiltonian):

$$
|\nu(t)|^{2}=\left|\int_{\sigma} d \mu_{0}(\epsilon) e^{-l \epsilon t}\right|^{2},
$$

where $\mu_{0}$ is the measure induced by the initial state $|\psi(0)\rangle$. Therefore its Fourier transform is the measure itself.

At this point it is important to stress that in the study of quantum walks it is often the case that the probability for the walker to be found at the initial position on the lattice is taken as a figure of merit to classify classical random and quantum walks in recursive and transient walks [47-49] regardless of the state of the coin. Here, however, we are interested in the return of the whole system to its initial state, including the state of the coin. Therefore, the spreading (localization) properties we shall refer to in the following are to be meant in the sense of spreading (localization) through the whole Hilbert space of the system (which is a tensor product of the Hilbert space of the walker's coin and position space) and not only in the physical space of the positions of the walker. A link between the two might be possible by reconstruction of the state of the 
coin, which might give useful information on the recurrence properties of the walker alone [50-52].

A second quantity we will explore is the Cesáro average (time average) of the echo, which is defined as

$$
\left\langle|\nu|^{2}\right\rangle_{T}=\frac{1}{T} \sum_{t=0}^{T-1}|v(t)|^{2} .
$$

Given that the survival amplitude in Eq. (14) is the Fourier transform of the measure, one can identify a singular continuous spectrum whenever the conditions [compare with Eq. (2) in Ref. [54] for the averaged correlation function of an observable $f(t)]$

$$
\begin{aligned}
\lim _{t \rightarrow \infty}|v(t)| \neq 0 & \text { not absolute continuous, } \\
\lim _{T \rightarrow \infty}\left\langle|v|^{2}\right\rangle_{T}=0 & \text { not pure point }
\end{aligned}
$$

are both satisfied. The first condition ensures the absence of an absolutely continuous part in the spectrum because, according to the Ruelle, Amrein-Georgescu, Enss (RAGE) theorem [46], $\lim _{t \rightarrow \infty} v(t)=0$ is a necessary condition for the presence of an absolutely continuous part in the spectrum. The second condition ensures the absence of the pure point spectrum due to Wiener's lemma (see Lemma 1.1 in [53]). Therefore the simultaneous occurrence of conditions in Eqs. (16a) and (16b) can be interpreted as the spectrum having only singular continuous parts.

A similar link between the singular continuous nature of energy spectra, the RAGE theorem, and the spreading properties of the walker has recently been investigated in Refs. [56,57]. In these works it was shown that the singular nature of the energy spectrum entails a power-law decay of the recurrence probability for the walker to be found in a bounded spatial region.

\section{A. Position dependent coin operations}

In the case of position dependent coin operations the corresponding DTQW is autonomous and therefore its asymptotic properties are directly related to the system's energy spectrum (and to the initial state). As in previous sections we shall consider an initial state such that the walker is localized at some site $\left|x=x_{0}\right\rangle$, however we will see that the asymptotic properties are largely independent of both the initial position of the walker and the initial coin state.

Since a state that is initially localized in real space is completely delocalized in momentum space, we expect it to have a nonvanishing overlap with most of the eigenstates of the evolution operator. Indeed, the peaks in the Fourier transforms $|\tilde{v}(u)|$ of the survival amplitude in Fig. 17 have values $L=2001, \theta_{1}=\pi / 4, \theta_{2}=\pi / 6$ and closely resemble those of the DOS shown in Fig. 4.

Examining $\tilde{v}(u)$ in more detail, we notice that in the periodic case a broad series of states (continuum) is occupied around the main peaks, whereas for the aperiodic arrangements a series of sharp peaks exists the positions of which are aligned with those of the corresponding DOS shown in Fig. 4. This is a hint of the singular nature of the measure induced by such an initial state which we expect to belong to the singular subspace of the Hilbert space [46]. In the following we will determine whether
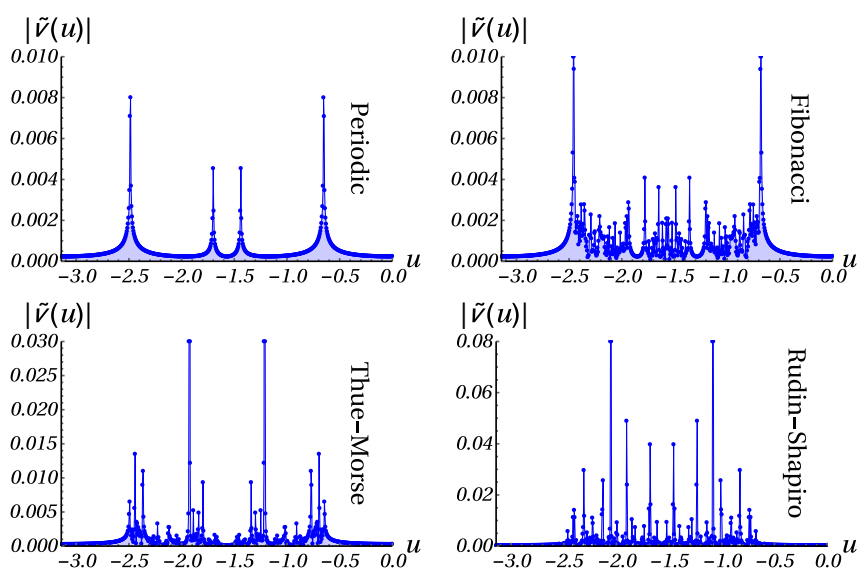

FIG. 17. Fourier transform $\tilde{v}(u)$ of the survival probability $v(t)$ for DTQWs with spatially inhomogeneous coins. The angles chosen are $\theta_{1}=\pi / 4, \theta_{2}=\pi / 6$, and the initial state is $|\psi(0)\rangle=\left|x_{0}=0\right\rangle \otimes$ $(|\uparrow\rangle+i|\downarrow\rangle) / \sqrt{2}$.

the measure induced by different initial states belongs to the pure point or singular continuous part.

In Fig. 18 we show the asymptotic values of $|v(t)|$ and the corresponding Cesáro averages, $\left\langle|v|^{2}\right\rangle_{T}$ for the different aperiodic sequences and for the particular initial state $|\psi(0)\rangle=$ $\left|x_{0}=0\right\rangle \otimes\left(\frac{|\uparrow\rangle+i|\downarrow\rangle}{\sqrt{2}}\right)$.

For the periodic case (not shown) $|v(t)|$ goes to zero and therefore, according to the RAGE theorem, the energy spectrum of the system has an absolutely continuous part, as it is to be expected. On the other hand for all aperiodic sequences $v(t)$ does not asymptotically vanish, implying that the corresponding energy spectra lack an absolutely continuous part. We notice that among all the Rudin-Shapiro sequence is the one which maintains the highest value at long times.
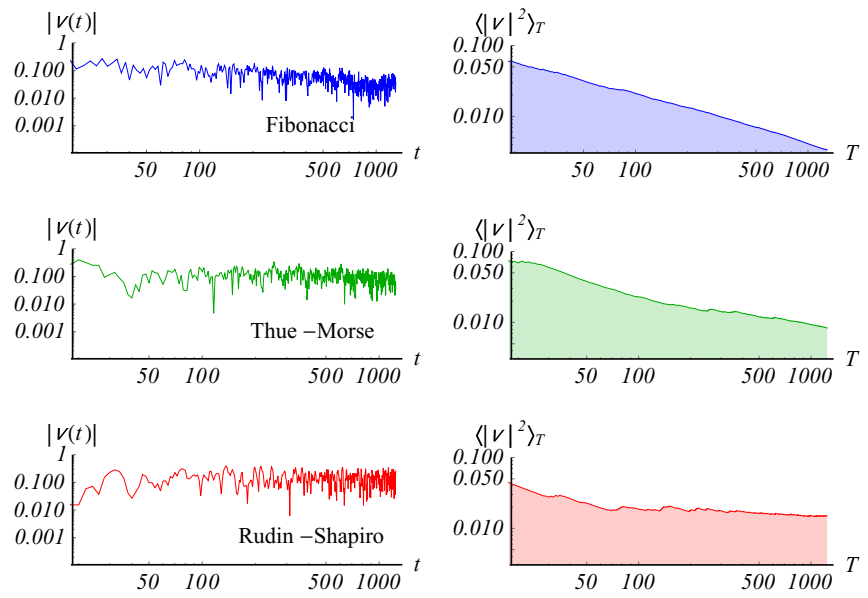

FIG. 18. Left: Absolute value of the survival amplitude $|v(t)|$ for different distributions of the coin operators in DTQWs with spatially aperiodically distributed coin operations. Right: Corresponding Cesáro averages of $|v(t)|^{2}$. All plots are in double logarithmic scale. Parameters are $L=2001, \theta_{1}=\pi / 4, \theta_{2}=\pi / 6$ and the initial state is $|\psi(0)\rangle=\left|x_{0}=0\right\rangle \otimes(|\uparrow\rangle+i|\downarrow\rangle) / \sqrt{2}$. 

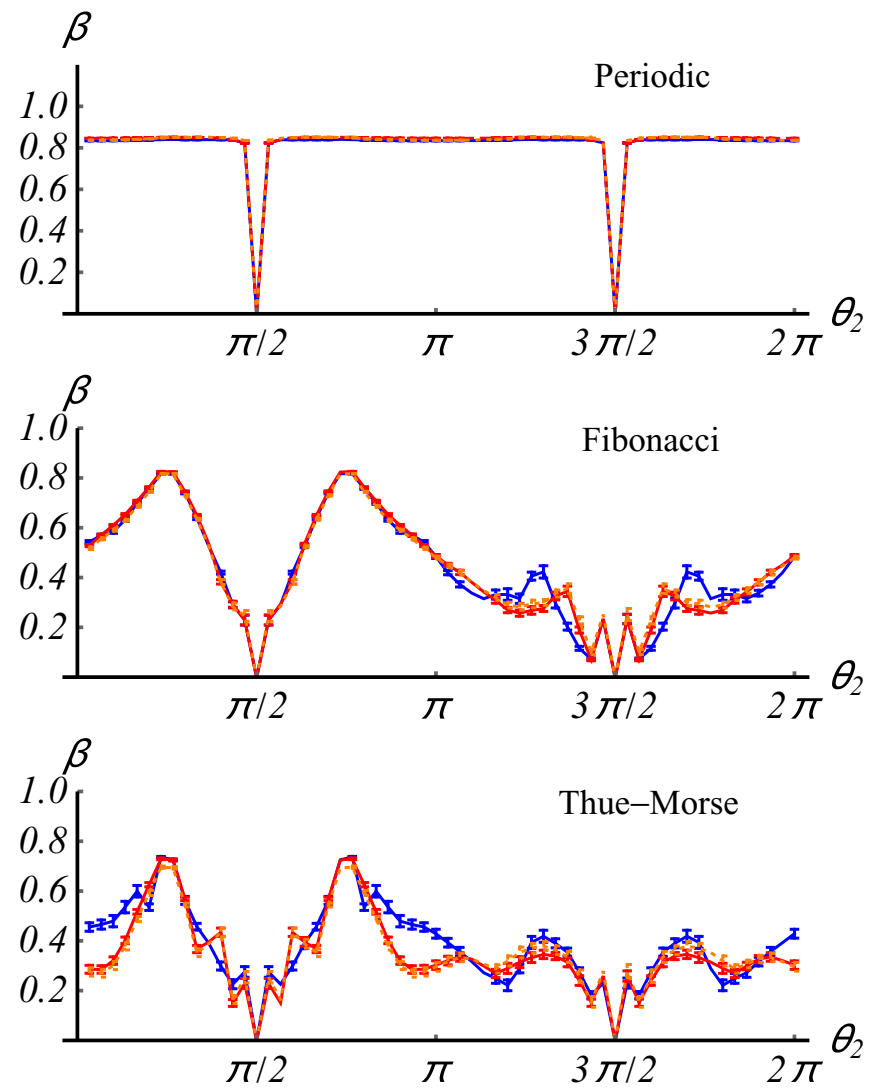

FIG. 19. Average value of the exponent $\beta$ (and relative error bars) describing the power-law decay of the Cesáro average at long times as a function of $\theta_{2}$. The average is obtained by fitting curves for 101 different initial positions of the walker on the lattice. The three curves refer to different system sizes and/or initial coin states: blue solid line, $L=1001$; red dashed line, $L=2001$ and the coin state is $\left(\frac{|\uparrow\rangle+i|\downarrow\rangle}{\sqrt{2}}\right)$ for both; orange dotted line, $L=2001$ and the coin initial state is $|\uparrow\rangle$.

The Cesáro average goes to zero algebraically, as shown in the double logarithmic plots, only for the Fibonacci and for the Thue-Morse cases. Together with the nonvanishing behavior of $|v(t)|$ at long times we expect these systems to have a spectrum with only singular continuous support. In the case of the Rudin-Shapiro sequence, the Cesáro average saturates to a constant value, suggesting the presence of a discrete part in the energy spectrum. This fact is in agreement with what is shown in Fig. 11 (top) where the walker remains localized at its initial position. We therefore expect the Rudin-Shapiro case to have a discrete energy spectrum and exponentially localized eigenstates. This behavior was also to be expected from the absolutely continuous nature of the diffraction spectrum of the Rudin-Shapiro case, resembling that of a disordered material where localization can be expected at the single particle level. In fact, this localization might be useful for applications such as quantum memories using DTQWs [55].

Similarly to the analysis performed for $\sigma(t)$ in Sec. V we have to consider different initial states for inhomogeneous DTQWs. We therefore considered different initial positions of the walker as well as different system sizes and different initial states of the coin. Assuming a power-law dependence $\langle v\rangle_{T} \propto T^{\beta}$, we show in Fig. 19 the exponent $\beta$ for the
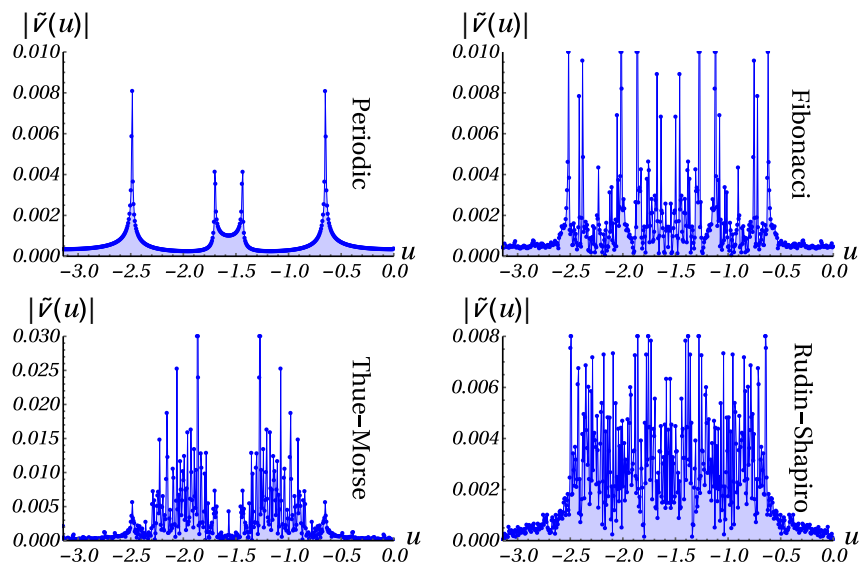

FIG. 20. Fourier transform $\tilde{v}(u)$ of the survival probability $v(t)$ for temporally inhomogeneous DTQWs. The angles chosen are $L=$ 2001, $\theta_{1}=\pi / 4, \theta_{2}=\pi / 6$ and the initial state is $|\psi(0)\rangle=\left|x_{0}=0\right\rangle \otimes$ $(|\uparrow\rangle+i|\downarrow\rangle) / \sqrt{2}$

periodic, the Fibonacci, and the Thue-Morse cases obtained for $N=101$ different initial positions of the walker around the center $x_{0}=0$. The error bars for each point are also reported. We can see that there is no significant dependence of the value of $\beta$ on the initial state of the coin (also confirmed by other cases not shown), however increasing the system size changes the value of $\beta$ and we assume it will eventually reach an asymptotic value. Regardless of its actual value, for what we are interested in here, it is the power-law dependence of the Cesáro average which tells us that for Fibonacci and Thue-Morse cases it will vanish at long enough times. We can therefore conclude that in these two cases the energy spectra have only singular continuous support. On the other hand by looking at the behavior of the echo and the corresponding Cesáro averages for different initial states and system sizes for inhomogeneous DTQWs with a Rudin-Shapiro sequence (all similar to the one shown in Fig. 18) we conclude that the Rudin-Shapiro sequence induces a discrete energy spectrum.

\section{B. Time dependent coin operations}

In the case of time dependent coin operations linking the asymptotic behavior to the spectral properties is slightly different than for the inhomogeneous case. This is due to the nonautonomous nature of the dynamics of the system, as the system is driven from the outside and therefore there is no well defined energy spectrum which we can refer to. On the other hand, as we have seen in Sec. IV, the energy spectrum of the system tends to some asymptotic spectrum after few iterations. It is therefore interesting to look at the asymptotic behavior and relate it to the spectrum of the asymptotic evolution operator. The Fourier transform of the survival amplitude $\tilde{v}(u)$ is shown in Fig. 20 and shares the same structure with the corresponding DOS, similar to the inhomogeneous case discussed above. However, the peaks in $\tilde{v}(u)$ are not similar to the ones in the corresponding DOS of the asymptotic evolution operator, which is shown in Fig. 7. In fact in the time dependent case $\tilde{v}(u)$ does not give information only on the measure induced by the initial state, but has a more complex structure the properties of which are beyond the scope of our paper. Nevertheless it is 

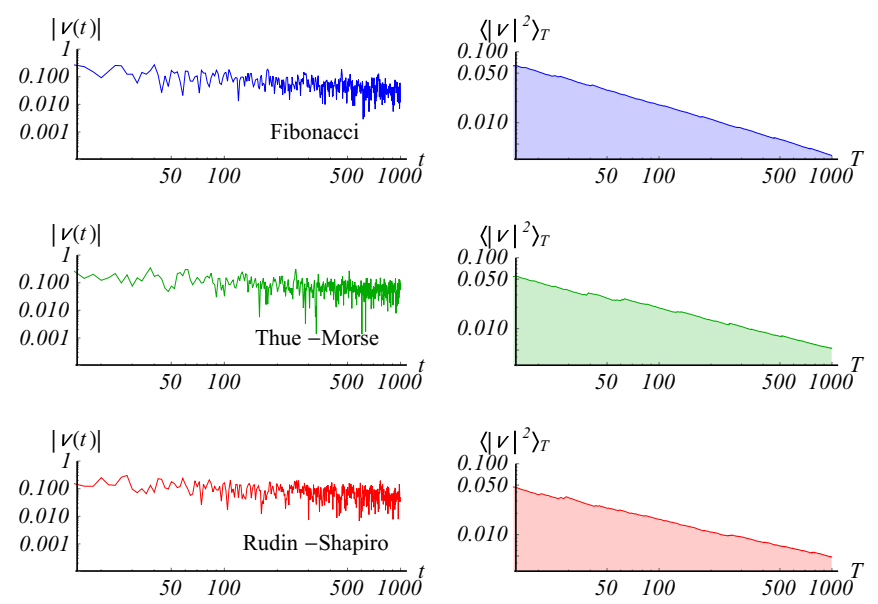

FIG. 21. Left: Absolute value of the survival amplitude $|v(t)|$ for different distributions of the coin operators in time dependent DTQWs. Right: Corresponding Cesáro averages of $|v(t)|^{2}$. All plots are in double logarithmic scale. Parameters are $L=2001, \theta_{1}=$ $\pi / 4, \theta_{2}=\pi / 6$ and the initial state is $|\psi(0)\rangle=\left|x_{0}=0\right\rangle \otimes(|\uparrow\rangle+$ $i|\downarrow\rangle) / \sqrt{2}$.

interesting to note the high fragmentation of the peaks for the aperiodic sequences which gives some evidence of singularity in the dynamics of the system. Taking this point of view it is therefore interesting to look again at the behavior of the survival amplitudes and their Cesáro averages.

From the left hand side of Fig. 21 we can see that the survival amplitudes do not go to zero for long times, allowing us to state that the corresponding energy spectra of the asymptotic evolution operators do not possess an absolutely continuous part. Looking at the Cesáro averages (right hand side of Fig. 21), we observe that the Fibonacci and the ThueMorse cases show again a power-law decay. Surprisingly, this time also the Rudin-Shapiro case shares this property. This difference in behavior between the inhomogeneous and the time dependent DTQWs was already visible from the spatial spreading of the walker reported in Fig. 11: for the case of the time dependent case the wave packet spreads further than in the case of the spatial Rudin-Shapiro distribution. We therefore conclude that for the time dependent case all considered aperiodic sequences induce a singular continuous energy spectrum in the asymptotic evolution operator as can be seen from Fig. 22 showing that the Cesáro average decays for all aperiodic sequences.

The time dependent cases addressed in our paper can also be seen from the point of view of the study of chaotic quantum systems. For instance, in the case of the Fibonacci temporal distribution of the coin operators, our findings can be linked to the emergence of a chaotic behavior in the dynamics of the walker generated by the trace map as the difference between the two coin operations increases. Indeed, in Ref. [27] it was shown that the trace map of the Fibonacci quantum walk for given initial conditions shows an increase in the chaotic behavior of the system. The power-law behavior is then ascribed to the system being trapped in a few of the (small) elliptic points left, which lead the system to temporarily localize, but from which it will eventually escape. For the Thue-Morse and Rudin-Shapiro cases an analysis in terms of
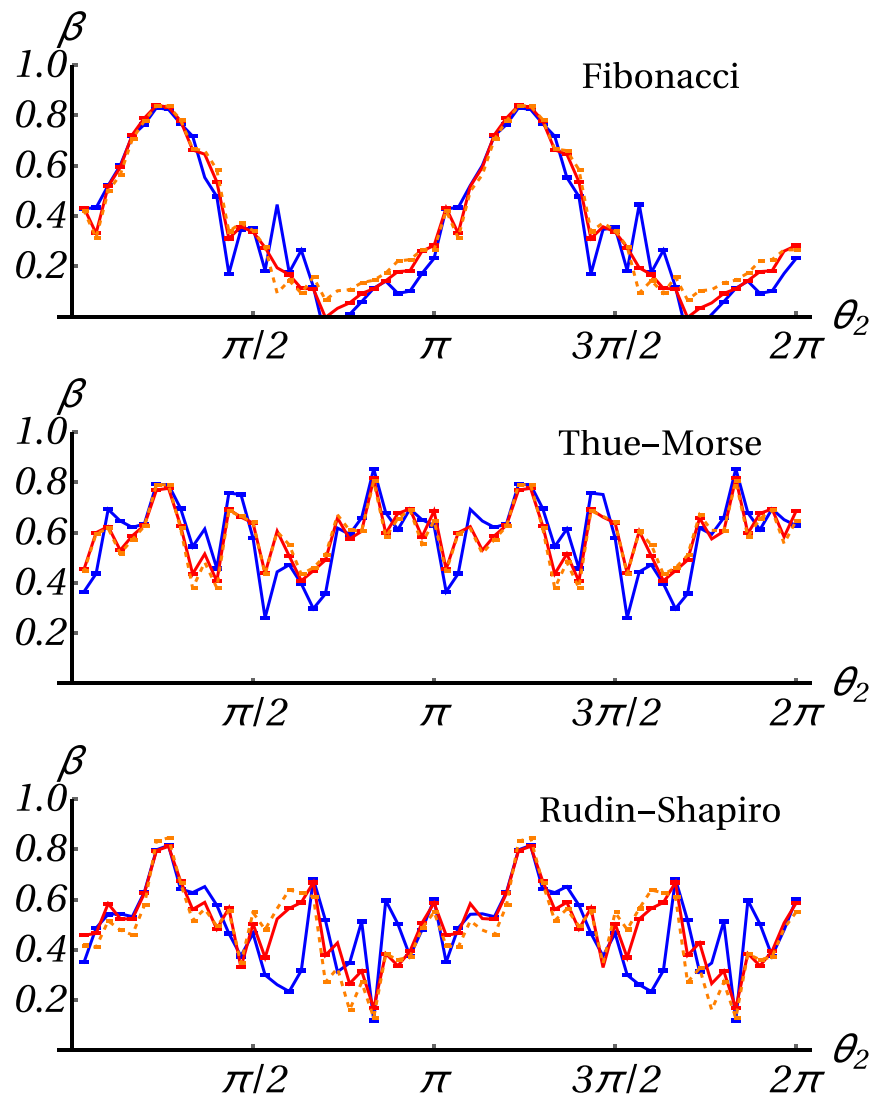

FIG. 22. Average value of the exponent $\beta$ (and relative error bars) describing the power-law decay of the Cesáro average at long times as a function of $\theta_{2}$. The average is obtained by fitting curves for 101 different initial positions of the walker on the lattice. The three curves refer to different system size and/or initial coin state: blue solid line, $L=1001$; red dashed line, $L=2001$ and the coin state is $\left(\frac{|\uparrow\rangle+i|\downarrow\rangle}{\sqrt{2}}\right)$ for both; orange dotted line, $L=2001$ and the coin initial state is $|\uparrow\rangle$.

maps and their relative attractors has not yet been done to the best of our knowledge but it would be interesting to look at it in more detail in order to understand whether the observed power-law behavior can be ascribed to the same mechanism and to link it to the role of the singular nature of the asymptotic energy spectra.

\section{CONCLUSIONS}

We have studied the dynamics of discrete-time quantum walks with different configurations of aperiodic sequences of position and time dependent coin operations and have investigated how the order of a given aperiodic sequence, as determined from its diffraction spectrum, plays a role in the dynamics of the walker. Specifically we have chosen three instances of aperiodic sequences, namely, the Fibonacci, Thue-Morse, and Rudin-Shapiro ones, which are known to have diffraction spectra with a pure point, singular continuous, and absolutely continuous spectrum, respectively. The pure point spectrum can be seen as the spectrum of a normal periodic crystal, the absolutely continuous one is similar to that of a completely disordered material, whereas the singular continuous one is peculiar of "critical" systems (meaning 
here one which is neither perfectly ordered nor completely disordered). We have explored the link between these types of energy spectra and the spreading of the walker and its asymptotic behavior.

We found that when the aperiodic sequence is used to distribute the action of two coins through the lattice, the Fibonacci and Thue-Morse cases show a superdiffusive behavior which is independent from the initial position of the walker and to some extent from the system size. The RudinShapiro sequence induces spatial localization of the walker, which can be understood from the absolutely continuous nature of the diffraction spectrum, which means the system closely resembles a disordered material. On the other hand this behavior is unusual because the Rudin-Shapiro sequence is derived from a well defined deterministic rule and therefore it possesses an intrinsic order.

The other case we have considered is that of aperiodic sequences used to perturb the system in time with coin operations which are the same throughout the whole lattice at a given time but change at each time step according to the chosen sequence. In this case we have observed spreading for all three sequences, although each of them with peculiar properties. We have also determined the nature of the energy spectra induced by the aperiodic sequences by looking at the survival amplitudes and their Cesáro averages (time averages). We have concluded that in the case of the inhomogeneous DTQW the Fibonacci and Thue-Morse sequences induce a singular continuous energy spectrum, whereas the RudinShapiro case induces a discrete one. On the other hand in the time dependent case the asymptotic evolution operator has a singular continuous energy spectrum for all considered aperiodic sequences.

\section{ACKNOWLEDGMENTS}

N.L.G. thanks F. Plastina for useful discussions. N.L.G. and L.D. acknowledge financial support by Ministero dell'Istruzione, Università e Ricerca (MIUR) through FIRB Project No. RBFR12NLNA002. N.L.G. acknowledges financial support by the European Union Collaborative project QuProCS (Grant No. 641277) and the Institute of Mathematical Sciences (IMS) of Chennai for hospitality. C.M.C. acknowledges support from Department of Science and Technology (DST) through Ramanujan Fellowship Grant No. SB/S2/RJN-192/2014. This work was supported by the Okinawa Institute of Science and Technology Graduate University. The authors are grateful to the anonymous referees for very useful comments on our paper during the review process.
[1] Y. Aharonov, L. Davidovich, and N. Zagury, Quantum random walks, Phys. Rev. A 48, 1687 (1993).

[2] E. Farhi and S. Gutmann, Quantum computation and decision trees, Phys. Rev. A 58, 915 (1998).

[3] S. E. Venegas-Andraca, Quantum walks: a comprehensive review, Quant. Info. Proc. 11, 1015 (2012).

[4] J. Kempe, Quantum random walks: an introductory overview, Contemp. Phys. 44, 307 (2003).

[5] A. M. Childs, Universal Computation by Quantum Walk, Phys. Rev. Lett. 102, 180501 (2009).

[6] N. B. Lovett, S. Cooper, M. Everitt, M. Trevers, and V. Kendon, Universal quantum computation using the discrete-time quantum walk, Phys. Rev. A 81, 042330 (2010).

[7] A. Schreiber, K. N. Cassemiro, V. Potocek, A. Gábris, I. Jex, and Ch. Silberhorn, Decoherence and Disorder in Quantum Walks: From Ballistic Spread to Localization, Phys. Rev. Lett. 106, 180403 (2011).

[8] C. M. Chandrashekar, Disordered-quantum-walk-induced localization of a Bose-Einstein condensate, Phys. Rev. A 83, 022320 (2011).

[9] T. Kitagawa, M. S. Rudner, E. Berg, and E. Demler, Exploring topological phases with quantum walks, Phys. Rev. A 82, 033429 (2010).

[10] D. A. Meyer, From quantum cellular automata to quantum lattice gases, J. Stat. Phys. 85, 551 (1996).

[11] G. Di Molfetta, M. Brachet, and F. Debbasch, Quantum walks as massless Dirac fermions in curved space-time, Phys. Rev. A 88, 042301 (2013).

[12] C. M. Chandrashekar, Two-component Dirac-like Hamiltonian for generating quantum walk on one-, twoand three-dimensional lattices, Scientific Reports 3, 2829 (2013).
[13] G. Di Molfetta, M. Brachet, and F. Debbasch, Quantum walks in artificial electric and gravitational fields, Physica A 397, 157 (2014).

[14] C. M. Chandrashekar, S. Banerjee, and R. Srikanth, Relationship between quantum walks and relativistic quantum mechanics, Phys. Rev. A 81, 062340 (2010).

[15] J. Du, H. Li, X. Xu, M. Shi, J. Wu, X. Zhou, and R. Han, Experimental implementation of the quantum random-walk algorithm, Phys. Rev. A 67, 042316 (2003).

[16] C. A. Ryan, M. Laforest, J. C. Boileau, and R. Laflamme, Experimental implementation of a discrete-time quantum random walk on an NMR quantum-information processor, Phys. Rev. A 72, 062317 (2005).

[17] H. Schmitz, R. Matjeschk, Ch. Schneider, J. Glueckert, M. Enderlein, T. Huber, and T. Schaetz, Quantum Walk of a Trapped Ion in Phase Space, Phys. Rev. Lett. 103, 090504 (2009).

[18] F. Zähringer, G. Kirchmair, R. Gerritsma, E. Solano, R. Blatt, and C. F. Roos, Realization of a Quantum Walk with One and Two Trapped Ions, Phys. Rev. Lett. 104, 100503 (2010).

[19] M. Karski, L. Förster, J. Choi, A. Steffen, W. Alt, D. Meschede, and A. Widera, Quantum walk in position space with single optically trapped atoms, Science 325, 174 (2009).

[20] M. Genske, W. Alt, A. Steffen, A. H. Werner, R. F. Werner, D. Meschede, and A. Alberti, Electric Quantum Walks with Individual Atoms, Phys. Rev. Lett. 110, 190601 (2013).

[21] B. Do, M. L. Stohler, S. Balasubramanian, D. S. Elliott, C. Eash, E. Fischbach, M. A. Fischbach, A. Mills, and B. Zwickl, Experimental realization of a quantum quincunx by use of linear optical elements, Opt. Soc. Amer. B 22, 499 (2005).

[22] M. A. Broome, A. Fedrizzi, B. P. Lanyon, I. Kassal, A. AspuruGuzik, and A. G. White, Discrete Single-Photon Quantum Walks with Tunable Decoherence, Phys. Rev. Lett. 104, 153602 (2010). 
[23] A. Nayak and A. Vishwanath, Quantum walk on the line, DIMACS Technical Report No. 2000-43, 2001 (unpublished).

[24] P. W. Anderson, Absence of diffusion in certain random lattices, Phys. Rev. 109, 1492 (1958).

[25] C. M. Chandrashekar, Disorder induced localization and enhancement of entanglement in one- and two-dimensional quantum walks, arXiv:1212.5984 (2012).

[26] P. Ribeiro, P. Milman, and R. Mosseri, Aperiodic Quantum Random Walks, Phys. Rev. Lett. 93, 190503 (2004).

[27] A. Romanelli, The Fibonacci quantum walk and its classical trace map, Physica A 388, 3985 (2009).

[28] C. Ampadu, Limit theorems for the Fibonacci quantum walk, arXiv:1108.5198 (2011).

[29] J. D. Joannopoulos, P. R. Villeneuve, and S. Fan, Photonic crystals: putting a new twist on light, Nature (London) 386, 143 (1997).

[30] Z. V. Vardeny, A. Nahata, and A. Agrawal, Optics of photonic quasicrystals, Nat. Photonics 7, 177 (2013).

[31] D. Shechtman, I. Blech, D. Gratias, and J. W. Cahn, Metallic Phase with Long-Range Orientational Order and no Translational Symmetry, Phys. Rev. Lett. 53, 1951 (1984).

[32] M. Schreiber et al., Observation of many-body localization of interacting fermions in a quasirandom optical lattice, Science 349, 842 (2015).

[33] J. Settino, N. Lo Gullo, A. Sindona, J. Goold, and F. Plastina, Signatures of the single-particle mobility edge in the groundstate properties of Tonks-Girardeau and noninteracting Fermi gases in a bichromatic potential, Phys. Rev. A 95, 033605 (2017).

[34] M. Kohmoto, C. Kadanoff, and C. Tang, Localization Problem in One Dimension: Mapping and Escape, Phys. Rev. Lett. 50, 1870 (1983).

[35] R. Merlin, K. Bajema, R. Clarke, F. Y. Juang, and P. K. Bhattacharya, Quasiperiodic GaAs-AlAs Heterostructures, Phys. Rev. Lett. 55, 1768 (1985).

[36] R. Riklund, M. Severin, and Y. Liu, The Thue-Morse aperiodic crystal, a link between the Fibonacci quasicrystal and the periodic crystal, Int. J. Mod. Phys. B 01, 121 (1987).

[37] M. S. Vasconcelos and E. L. Albuquerque, Transmission fingerprints in quasiperiodic dielectric multilayers, Phys. Rev. B 59, 11128 (1999).

[38] B. L. Burrows and K. W. Sulston, Measurement of disorder in non-periodic sequences, J. Phys. A 24, 3979 (1991).

[39] V. Berthe, Conditional entropy of some automatic sequences, J. Phys. A 27, 7993 (1994).

[40] L. S. Levitov, Localization-Delocalization Transition for OneDimensional Alloy Potentials, Europhys. Lett. 7, 343 (1988).
[41] J. M. Luck, Cantor spectra and scaling of gap widths in deterministic aperiodic systems, Phys. Rev. B 39, 5834 (1989).

[42] A. Suto, Singular continuous spectrum on a cantor set of zero Lebesgue measure for the Fibonacci Hamiltonian, J. Stat. Phys 56, 525 (1989).

[43] J.-M. Gambaudo and P. Vignolo, Brillouin zone labelling for quasicrystals, New J. Phys. 16, 043013 (2014).

[44] N. Lo Gullo, L. Vittadello, M. Bazzan, and L. Dell'Anna, Equivalence classes of Fibonacci lattices and their similarity properties, Phys. Rev. A 94, 023846 (2016).

[45] C. M. Chandrashekar, R. Srikanth, and R. Laflamme, Optimizing the discrete time quantum walk using a $\mathrm{SU}(2)$ coin, Phys. Rev. A 77, 032326 (2008).

[46] Y. Last, Quantum dynamics and decompositions of singular continuous spectra, J. Funct. An. 142, 406 (1996).

[47] M. Štefaňáck, I. Jex, and T. Kiss, Recurrence and Pólya Number of Quantum Walks, Phys. Rev. Lett. 100, 020501 (2008).

[48] M. Štefaňáck, I. Jex, and T. Kiss, Recurrence of biased quantum walks on a line, New J. Phys. 11, 043027 (2009).

[49] C. M. Chandrashekar, Fractional recurrence in discrete-time quantum walk, Open Physics 8, 979 (2010).

[50] J. Goold, T. Fogarty, N. Lo Gullo, M. Paternostro, and Th. Busch, Orthogonality catastrophe as a consequence of qubit embedding in an ultracold Fermi gas, Phys. Rev. A 84, 063632 (2011).

[51] P. Haikka, J. Goold, S. McEndoo, F. Plastina, and S. Maniscalco, Non-Markovianity, Loschmidt echo, and criticality: a unified picture, Phys. Rev. A 85, 060101 (2012).

[52] A. Sindona, J. Goold, N. Lo Gullo, S. Lorenzo, and F. Plastina, Orthogonality Catastrophe and Decoherence in a TrappedFermion Environment, Phys. Rev. Lett. 111, 165303 (2013).

[53] M. Queffélec, Substitution Dynamical Systems: Spectral Analysis, 2nd ed., Lecture Notes in Mathematics Vol. 1294 (SpringerVerlag, Berlin, 2010).

[54] A. S. Pikovsky, M. A. Zaks, U. Feudel, and J. Kurths, Singular continuous spectra in dissipative dynamics, Phys. Rev. E 52, 285 (1995).

[55] C. M. Chandrashekar and Th. Busch, Localized quantum walks as secured quantum memory, Europhys. Lett. 110, 10005 (2015).

[56] D. Damanik, J. Fillman, and D. C. Ong, Spreading estimates for quantum walks on the integer lattice via power-law bounds on transfer matrices, J. Math. Pures et Appliquées 105, 293 (2015).

[57] J. Fillman and D. C. Ong, Purely singular continuous spectrum for limit-periodic CMV operators with applications to quantum walks, J. Func. Analysis 272, 5107 (2017). 